\title{
ADDITIVE VERSUS ABELIAN 2-REPRESENTATIONS OF FIAT 2-CATEGORIES
}

\author{
VOLODYMYR MAZORCHUK AND VANESSA MIEMIETZ
}

\begin{abstract}
We study connections between additive and abelian 2-representations of fiat 2-categories, describe combinatorics of 2-categories in terms of multisemigroups and determine the annihilator of a cell 2-representation. We also describe, in detail, examples of fiat 2-categories associated to $\mathfrak{s l}_{2}$ categorification in the sense of Chuang and Rouquier, and 2-categorical analogues of Schur algebras.
\end{abstract}

\section{INTRODUCTION AND DESCRIPTION OF THE RESULTS}

2-categories appear naturally in the study of "categorifications" of various classical mathematical objects, see for example [Ro1, Ro2, MM]. Natural representations of 2-categories are called 2-representations and represent 2-categories using functorial actions on some classical categories, for example additive or abelian categories. In our previous paper $[\mathrm{MM}]$ we defined a class of fiat 2-categories which we believe is a proper 2-categorical analogue of finite dimensional associative algebras with involution and started the project of understanding the 2-representation theory of such 2-categories. The main result of $[\mathrm{MM}]$ is the construction and study of what we call cell 2-representations, which we believe is a sensible 2-categorical analogue of simple modules.

The present paper, on the one hand, continues and extends the study from [MM] and, on the other hand, proposes an approach to these questions from a slightly different perspective. The paper $[\mathrm{MM}]$ mostly studies 2 -representations of fiat categories in abelian categories. In the present paper we go back to the original point of view of Rouquier, see [Ro2], and try to represent fiat categories using additive categories instead. The advantage is that many constructions are simplified, the disadvantage is that there is not much one can do with additive 2-representations. To combine the advantages of both approaches we connect additive and abelian categorifications via a 2 -functor, called the abelianization 2 -functor. As an immediate consequence we obtain natural constructions for many new 2-representations as follows: given a fiat 2-category $\mathscr{C}$ and an additive 2-representation $\mathbf{M}$ of $\mathscr{C}$, one can abelianize $\mathbf{M}$ and then find many additive subrepresentations inside this abelian 2-representation by taking additive closures of any collection of objects stable (up to isomorphism) under the action of $\mathscr{C}$.

Let us briefly describe the content of the paper. In Section 2 we collect all necessary preliminaries on 2-categories, 2-representation and decategorifications together with some classical examples. Section 3 describes the combinatorics of 2-categories using the language of multisemigroups. A multisemigroup is a "semigroup" with a multivalued operation. Isomorphism classes of 1-morphisms in a 2-category form in a natural way a multisemigroup with respect to the operation induced by the horizontal composition. Similarly to classical semigroup theory, on each multisemigroup one can define Green's equivalence relations which control when two elements 
of a (multi)semigroup generate the same principal left-, right- or two-sided ideals. We explain that the latter coincide with the notions of left-, right- and two-sided cells for 2-categories as introduced in [MM].

In Section 4 we discuss the connection between additive and abelian 2-representations as described above. We define principal additive 2-representations, the abelianization 2-functor and explain how to find additive 2-subrepresentations in abelian 2-representations. As an application, we reformulate the construction of cell 2-representations of fiat categories from [MM] using this machinery. Section 5 is dedicated to the comparison of various classes of 2 -representations. We show that every abelian representation of a fiat 2-category is equivalent to the abelianization of the additive 2-subrepresentation given by the subcategory of projective objects and also extend the comparison result for cell 2-representations from $[\mathrm{MM}]$ to the additive setup.

In Section 6 we study annihilators of 2-representations, in particular, those of cell 2 -representations. In $[\mathrm{MM}]$ we show that cell 2-representations of fiat 2-categories have properties similar to those of simple representations of associative algebras. Here we add another property, formulated below, which says that the image of a fiat 2 -category on a cell 2-representation is "simple" in the sense that any nontrivial 2-ideal necessarily annihilates the cell 2-representation. Our main result is the following:

Theorem 1. Let $\mathcal{J}$ be a two-sided cell in a fiat 2-category $\mathscr{C}$ and $\mathcal{L}$ be a left cell in $\mathcal{J}$. Then the "image" 2-category $\mathscr{C} / \operatorname{Ker}\left(\mathbf{C}_{\mathcal{L}}\right)$ of the cell 2-representation $\mathbf{C}_{\mathcal{L}}$ is $\mathcal{J}$-simple.

Using Theorem 1, we propose an alternative construction for cell 2-representations by considering "simple" quotients of fiat categories associated to two-sided cells.

Finally, in Section 7 we describe, in detail, examples of fiat 2-categories associated to $\mathfrak{s l}_{2}$-categorification in the sense of Chuang and Rouquier (see $[\mathrm{CR}]$ ), and also 2categorical analogues of Schur algebras. We show that both of these examples have particularly nice combinatorial properties and hence all of our results from [MM] and the present paper are fully applicable. We complete the paper with one general construction for fiat categories inspired by the restriction of projective functors to parabolic blocks of the BGG category $\mathcal{O}$.

Acknowledgment. A substantial part of the paper was written during the visit, supported by ERC grant PERG07-GA-2010-268109, of the first author to the University of East Anglia, whose hospitality is gratefully acknowledged. The first author is also partially supported by the Swedish Research Council and the Royal Swedish Academy of Sciences. We thank the referee for helpful comments.

\section{2-CATEGORIES AND 2-REPRESENTATIONS}

2.1. 2-categories. If not stated otherwise, all categories considered in this paper are assumed to be small. We denote by Cat the locally small category of small categories, which is monoidal with respect to the cartesian product. By a 2-category we mean a category enriched over Cat. In other words, a 2-category $\mathscr{C}$ consists of the following data: a set $\mathscr{C}$ of objects; for every $i, j \in \mathscr{C}$ a small category $\mathscr{C}(i, j)$ of morphisms from $i$ to $j$ (objects of $\mathscr{C}(i, j)$ are called 1-morphisms of $\mathscr{C}$ while morphisms of $\mathscr{C}(i, j)$ are called 2-morphisms of $\mathscr{C})$; the identity 1-morphism 
$\mathbb{1}_{i} \in \mathscr{C}(\mathrm{i}, \mathrm{i})$ for all $\mathrm{i}$; and functorial compositions $\mathscr{C}(\mathrm{j}, \mathrm{k}) \times \mathscr{C}(\mathrm{i}, \mathrm{j}) \rightarrow \mathscr{C}(\mathrm{i}, \mathrm{k})$; which satisfy the strict versions of the usual axioms.

We retain the notational conventions from $[\mathrm{MM}]$. For a 2-category $\mathscr{C}$, objects of $\mathscr{C}$ will be denoted by i, j and so on; 1-morphisms of $\mathscr{C}$ will be called F, G and so on; 2 -morphisms of $\mathscr{C}$ will be written $\alpha, \beta$ and so on. The identity 2-endomorphism of a 1-morphism $\mathrm{F}$ will be denoted $\mathrm{id}_{\mathrm{F}}$. Composition of 1-morphisms will be denoted by $\circ$, horizontal composition of 2-morphisms will be denoted by $\circ_{0}$ and vertical composition of 2-morphisms will be denoted by $\circ_{1}$. We often abbreviate $\operatorname{id}_{\mathrm{F}} \circ_{0} \alpha$ and $\alpha \circ_{0} \operatorname{id}_{\mathrm{F}}$ by $\mathrm{F}(\alpha)$ and $\alpha_{\mathrm{F}}$, respectively. To avoid the cumbersome notation $\mathscr{C}(\mathrm{i}, \mathrm{j})(\mathrm{F}, \mathrm{G})$ we will write $\operatorname{Hom}_{\mathscr{C}(i, j)}(\mathrm{F}, \mathrm{G})$ instead.

2.2. Fiat categories. Let $\mathbb{k}$ be a field. A 2 -category $\mathscr{C}$ is called fiat (over $\mathbb{k}$ ) provided that the following conditions are satisfied:

- $\mathscr{C}$ has finitely many objects;

- every $\mathscr{C}(i, j)$ is a fully additive $\mathbb{k}$-linear category with finitely many indecomposable objects up to isomorphism;

- horizontal compositions are additive and $\mathbb{k}$-linear;

- every $\mathbb{k}$-linear space $\operatorname{Hom}_{\mathscr{C}_{(i, j)}}(\mathrm{F}, \mathrm{G})$ is finite dimensional;

- all 1-morphisms $\mathbb{1}_{i}$ are indecomposable;

- $\mathscr{C}$ has a weak object preserving involutive anti-autoequivalence $*$;

- $\mathscr{C}$ has adjunction morphisms, that is for any $i, j \in \mathscr{C}$ and any 1-morphism $\mathrm{F} \in \mathscr{C}(\mathrm{i}, \mathrm{j})$ there exist 2-morphisms $\alpha: \mathrm{F} \circ \mathrm{F}^{*} \rightarrow \mathbb{1}_{\mathrm{j}}$ and $\beta: \mathbb{1}_{i} \rightarrow \mathrm{F}^{*} \circ \mathrm{F}$ such that $\alpha_{\mathrm{F}} \circ_{1} \mathrm{~F}(\beta)=\mathrm{id}_{\mathrm{F}}$ and $\mathrm{F}^{*}(\alpha) \circ_{1} \beta_{\mathrm{F}^{*}}=\operatorname{id}_{\mathrm{F}^{*}}$.

Example 2. Let $A=A_{1} \oplus A_{2} \oplus \cdots \oplus A_{k}$ be a finite dimensional associative $\mathbb{k}$ algebra such that each $A_{i}$ is connected and not simple and the $A_{i}$ are pairwise non-isomorphic. Assume further that every $A_{i}$ is weakly symmetric (i.e. it is selfinjective and the top of each indecomposable projective is isomorphic to the socle). For $i=1, \ldots, k$ fix some small category $\mathcal{C}_{i}$, equivalent to $A_{i}$-mod. A projective functor from $\mathcal{C}_{i}$ to $\mathcal{C}_{j}$ is a functor isomorphic to tensoring with a projective $A_{j} A_{i^{-}}$ bimodule. Let $\mathscr{C}_{A}$ be the 2-category with $k$ objects $\mathcal{C}_{i}, i=1, \ldots, k ; 1$-morphisms being all projective functors between objects together with all endofunctors of objects isomorphic to the corresponding identity functors; and 2-morphisms being all natural transformations of functors. Then $\mathscr{C}_{A}$ is a fiat 2-category, see [MM, Subsection 7.3].

Example 3. Let $\mathfrak{g}$ be a semi-simple complex finite dimensional Lie algebra with a fixed triangular decomposition $\mathfrak{g}=\mathfrak{n}_{-} \oplus \mathfrak{h} \oplus \mathfrak{n}_{+}$, let $W$ be the Weyl group of $\mathfrak{g}$ and $\mathrm{C}_{W}$ the coinvariant algebra associated to the action of $W$ on $\mathfrak{h}^{*}$. For a simple reflection $s \in W$ let $\mathrm{C}_{W}^{s}$ denote the subalgebra of $s$-invariants in $\mathrm{C}_{W}$. For $w \in W$ fix some reduced expression $w=s_{1} \cdot s_{2} \cdots s_{k}$ and define the $\mathrm{C}_{W}{ }^{-} \mathrm{C}_{W}$-bimodule

$$
\hat{B}_{w}:=\mathrm{C}_{W} \otimes_{\mathrm{C}_{W}^{s_{1}}} \mathrm{C}_{W} \otimes_{\mathrm{C}_{W}^{s_{2}}} \cdots \otimes_{\mathrm{C}_{W}^{s_{k}}} \mathrm{C}_{W}
$$

Define $B_{e}:=\mathrm{C}_{W}$, and for $w \neq e$ define $B_{w}$ inductively (with respect to the length of $w)$ as the unique indecomposable direct summand of $\hat{B}_{w}$ that is not isomorphic to something already defined. The $\mathrm{C}_{W}-\mathrm{C}_{W}$-bimodule $B_{w}$ is called the Soergel bimodule associated with $w$ (see [So]). Up to isomorphism it does not depend on the choice of a reduced expression for $w$. Let $\mathcal{A}$ be some small category equivalent to $\mathrm{C}_{W}$-mod. Let $\mathscr{S}_{\mathfrak{g}}$ be the 2 -category with one object $\mathcal{A}$; 1 -morphisms being all endofunctors of 
$\mathcal{A}$ isomorphic to tensoring with direct sums of Soergel bimodules; and 2-morphisms being all natural transformations of functors. Then $\mathscr{S}_{\mathfrak{g}}$ is a fiat 2-category, see [MM, Subsection 7.1], the so-called 2-category of Soergel bimodules.

From now on, if not explicitly stated otherwise, $\mathscr{C}$ is always assumed to be a fiat 2-category.

2.3. 2-representations. Denote by $\mathfrak{A}_{\mathbb{k}}$ the locally small 2 -subcategory of Cat defined as follows: objects of $\mathfrak{A}_{\mathbb{k}}$ are small fully additive $\mathbb{k}$-linear categories, 1morphisms are additive $\mathbb{k}$-linear functors and 2-morphisms are natural transformations of functors. Denote by $\mathfrak{A}_{\mathbb{k}}^{f}$ the full subcategory of $\mathfrak{A}_{\mathbb{k}}$ containing all small fully additive $\mathbb{k}$-linear categories which have a finite number of indecomposable objects up to isomorphism and finite dimensional spaces of morphisms. Finally, denote by $\mathfrak{R}_{\mathbb{k}}$ the full subcategory of $\mathfrak{A}_{\mathbb{k}}$ containing all small fully additive $\mathbb{k}$-linear categories that are equivalent to module categories of finite dimensional associative $\mathbb{k}$-algebras.

Given a 2-category $\mathscr{C}$, a 2-representation of $\mathscr{C}$ is a 2 -functor from $\mathscr{C}$ to one of the 2-categories $\mathfrak{A}_{\mathbb{k}}, \mathfrak{A}_{\mathbb{k}}^{f}$ or $\mathfrak{R}_{\mathbb{k}}$. Representations of $\mathscr{C}$ in $\mathfrak{A}_{\mathbb{k}}, \mathfrak{A}_{\mathbb{k}}^{f}$ or $\mathfrak{R}_{\mathbb{k}}$ are called additive, finitary and abelian (or of additive, finitary or abelian type), respectively. Together with 2-natural transformations and modifications, additive, finitary and abelian representations of $\mathscr{C}$ form 2 -categories $\mathscr{C}$-amod, $\mathscr{C}$-afmod and $\mathscr{C}$-mod, respectively. We refer the reader to $[\mathrm{McL}, \mathrm{Ma}, \mathrm{MM}]$ for more details on 2-functors, 2-natural transformations and modifications.

Example 4. Both the 2-category $\mathscr{C}_{A}$ from Example 2 and the 2-category $\mathscr{S}_{\mathfrak{g}}$ from Example 3 are defined via the corresponding defining representation (given by the identity maps on all ingredients).

2.4. Decategorification. The decategorification of a fiat 2-category $\mathscr{C}$ is a category (i.e. a 1-category) $[\mathscr{C}]$ defined as follows: $[\mathscr{C}]$ has the same objects as $\mathscr{C}$; for $i, j \in[\mathscr{C}]$ the morphism space $[\mathscr{C}](i, j)$ is defined as the split Grothendieck group $[\mathscr{C}(i, j)]$ of the additive category $\mathscr{C}(i, j)$; the composition in $[\mathscr{C}]$ is induced by the composition in $\mathscr{C}$ (note that the latter is biadditive).

Given an additive 2-representations $\mathbf{M}$ of $\mathscr{C}$, the decategorification of $\mathbf{M}$ is a functor $[\mathbf{M}]$ from $[\mathscr{C}]$ to the category $\mathbf{A b}$ of abelian groups defined as follows: for $i \in[\mathscr{C}]$ the abelian group $[\mathbf{M}](\mathbf{i})$ is defined as the split Grothendieck group of $\mathbf{M}(\mathbf{i})$ and for any 1-morphism $\mathrm{F} \in \mathscr{C}(i, j)$, the action of the class $[F] \in[\mathscr{C}(i, j)]$ on $[\mathbf{M}](i)$ is induced by the functorial action of $\mathrm{F}$ on $\mathbf{M}(\mathrm{i})$ (note that the latter functor is additive). In this way, the decategorification of a 2-representation of $\mathscr{C}$ is a representation of $[\mathscr{C}]$. This gives rise to a functor from the category of additive 2-representations of $\mathscr{C}$ (with modifications forgotten) to the category of representations of $[\mathscr{C}]$ in Ab.

As $\mathscr{C}$ is fiat, every 1-morphism of $\mathscr{C}$ acts as an exact functor on any abelian 2 -representation of $\mathscr{C}$. Hence one similarly defines the decategorification of an abelian 2-representation of $\mathscr{C}$ using the usual Grothendieck group of an abelian category.

2.5. Equivalent 2-representations. Two 2-representations $\mathbf{M}$ and $\mathbf{N}$ of $\mathscr{C}$ are called elementary equivalent provided that there exists either a 2-natural transformation $\Phi: \mathbf{M} \rightarrow \mathbf{N}$ or a 2-natural transformation $\Phi: \mathbf{N} \rightarrow \mathbf{M}$ such that $\Phi_{\mathrm{i}}$ is an equivalence for any $i \in \mathscr{C}$. Unfortunately, we do not know whether the binary 
relation of elementary equivalence for 2 -representations is symmetric. Therefore to get a sensible notion of equivalent 2-representations given by a genuine equivalence relation, we call two 2-representations $\mathbf{M}$ and $\mathbf{N}$ of $\mathscr{C}$ equivalent provided that there is a sequence $\mathbf{M}_{1}, \mathbf{M}_{2}, \ldots, \mathbf{M}_{k}$ of 2-representations of $\mathscr{C}$ such that $\mathbf{M}_{1}=\mathbf{M}$, $\mathbf{M}_{k}=\mathbf{N}$ and for any $i=1,2, \ldots, k-1$ the 2-representations $\mathbf{M}_{i}$ and $\mathbf{M}_{i+1}$ are elementary equivalent. Note that equivalent 2-representations of $\mathscr{C}$ descend to isomorphic representations of $[\mathscr{C}]$.

Two 2-representations $\mathbf{M}$ and $\mathbf{N}$ of $\mathscr{C}$ are called isomorphic provided that there exists either a 2-natural transformation $\Phi: \mathbf{M} \rightarrow \mathbf{N}$ such that $\Phi_{i}$ is an isomorphism for any $i \in \mathscr{C}$. In this case the inverse isomorphism $\Phi^{-1}: \mathbf{N} \rightarrow \mathbf{M}$ is a 2-natural transformation as well.

\section{Combinatorics of Additive 2-CATEgories}

Before coming to 2-representations, we first address the question of internal structure of 2-categories. In this section we describe the multisemigroup approach to the combinatorics of additive $\mathbb{k}$-linear 2-categories. This is inspired by [Vi].

3.1. Multisemigroups. For a set $X$ we will denote by $\mathcal{B}(X)$ the set of all subsets of $X$ (the Boolean of $X$ ). For $x \in X$ we will identify the element $x$ with the subset $\{x\} \in \mathcal{B}(X)$.

A multisemigroup is a pair $(S, *)$ consisting of a set $S$ and a multivalued operation $*: S \times S \rightarrow \mathcal{B}(S)$ (written $(a, b) \mapsto a * b$ ), which is assumed to be associative in the sense that for all $a, b, c \in S$ one has

$$
\bigcup_{t \in a * b} t * c=\bigcup_{s \in b * c} a * s
$$

For example, any semigroup is a multisemigroup. Many other examples of multisemigroups can be found in [Vi], we just give one more in Example 5 below.

A unit of a multisemigroup $(S, *)$ is an element $e \in S$ such that $a * e=e * a=a$. If a multisemigroup has a unit, this unit is unique. If a multisemigroup $(S, *)$ does not have a unit, one can formally adjoin it by considering the multisemigroup $\left(S^{1}, \circledast\right)$, where $S^{1}=S \cup\{1\}$ for some $1 \notin S$, and defining the operation $\circledast$ for $a, b \in S^{1}$ as follows:

$$
a \circledast b= \begin{cases}a, & b=1 ; \\ b, & a=1 ; \\ a * b, & \text { otherwise. }\end{cases}
$$

If $(S, *)$ has a unit, we set $\left(S^{1}, \circledast\right)=(S, *)$.

Example 5. Consider the set $\mathbb{Z}_{+}=\{0,1,2,3, \ldots\}$ of all non-negative integers and for $m, n \in \mathbb{Z}_{+}$define

$$
m \diamond n=\left\{x \in \mathbb{Z}_{+}:|m-n| \leq x \leq m+n, x \equiv m+n \bmod 2\right\} .
$$

This makes $\left(\mathbb{Z}_{+}, \diamond\right)$ into a multisemigroup. The element 0 is the unit element of $\left(\mathbb{Z}_{+}, \diamond\right)$.

Any multisemigroup $(S, *)$ induces a semigroup structure on $\mathcal{B}(S)$ by extending the operation $*$ to the whole of $\mathcal{B}(S)$ as follows (here $X, Y \in \mathcal{B}(S))$ :

$$
X * Y=\bigcup_{x \in X, y \in Y} x * y .
$$

In what follows we will often use this extension of $*$. 
3.2. Green's relations in multisemigroups. Here we define analogues of the classical Green's relations for semigroups (see [Gr]) in our more general setup of multisemigroups. Let $(S, *)$ be a multisemigroup. For $a, b \in S$ we will write $a \leq_{L} b$, $a \leq_{R} b$ or $a \leq_{J} b$ in the cases $S^{1} * b \subset S^{1} * a, b * S^{1} \subset a * S^{1}$ and $S^{1} * b * S^{1} \subset S^{1} * a * S^{1}$, respectively. The partial pre-order relations $\leq_{L}, \leq_{R}$ and $\leq_{J}$ will be called the left, right and two-sided orders, respectively. Equivalence classes $\sim_{L}, \sim_{R}$ and $\sim_{J}$ of these relations will be called the left, right and two-sided cells, respectively. If $S$ is a semigroup, then left, right and two-sided cells coincide with Green's $\mathcal{L}$-, $\mathcal{R}$ - and $\mathcal{J}$-classes, respectively.

Example 6. For the multisemigroup $\left(\mathbb{Z}_{+}, \diamond\right)$ in Example 5 the whole multisemigroup is the unique cell, which is both left, right and two-sided at the same time.

3.3. Multisemigroup of a 2-category. For this subsection we just assume that $\mathscr{C}$ is small and fully additive (that is $\mathscr{C}(i, j)$ is additive for all $i$ and $j$ ) and that the composition bifunctor is biadditive. Denote by $\mathcal{S}(\mathscr{C})$ the set of isomorphism classes of indecomposable 1-morphisms in $\mathscr{C}$ with an element 0 added. For indecomposable 1-morphisms $\mathrm{F}$ and $\mathrm{G}$ let $[\mathrm{F}]$ and $[\mathrm{G}]$ denote the corresponding classes in $\mathcal{S}(\mathscr{C})$ and set

$$
[\mathrm{F}] *[\mathrm{G}]= \begin{cases}0, & \mathrm{~F} \circ \mathrm{G} \text { is undefined; } \\ 0, & \mathrm{~F} \circ \mathrm{G}=0 ; \\ \{[\mathrm{H}] \in \mathcal{S}(\mathscr{C}): \mathrm{H} \text { is a summand of } \mathrm{F} \circ \mathrm{G}\}, & \text { otherwise. }\end{cases}
$$

Associativity of horizontal composition in $\mathscr{C}$ implies that $\mathcal{S}(\mathscr{C})$ becomes a multisemigroup, which we will call the multisemigroup of $\mathscr{C}$. In case $[\mathrm{F}] *[\mathrm{G}] \neq 0$ for all $[\mathrm{F}]$ and $[\mathrm{G}]$ one can consider the multisubsemigroup $\mathcal{S}^{\prime}(\mathscr{C}):=\mathcal{S}(\mathscr{C}) \backslash\{0\}$ of $\mathcal{S}^{\prime}(\mathscr{C})$, which we will call the reduced multisemigroup of $\mathscr{C}$.

Example 7. Let $\mathscr{C}_{\mathfrak{s l}_{2}}$ denote the 2-category with one object i such that $\mathscr{C}_{\mathfrak{s l}_{2}}(\mathbf{i}, \mathbf{i})$ is a small category equivalent to the category of finite dimensional $\mathfrak{s l}_{2}(\mathbb{C})$-modules and such that the composition in $\mathscr{C}_{\mathfrak{s l}_{2}}$ is induced by the tensor product of $\mathfrak{s l}_{2}(\mathbb{C})$ modules. Mapping an $n+1$-dimensional simple $\mathfrak{s l}_{2}(\mathbb{C})$-module to $n$ identifies $\mathcal{S}^{\prime}(\mathscr{C})$ with $\mathbb{Z}_{+}$. The classical Clebsh-Gordon rule for $\mathfrak{s l}_{2}(\mathbb{C})$ implies that $\mathcal{S}^{\prime}(\mathscr{C})$ is isomorphic to the multisemigroup $\left(\mathbb{Z}_{+}, \diamond\right)$ from Example 5 .

Remark 8. One can also consider the natural generalization of multisemigroups using multisets (such objects perhaps should be then called multimultisemigroups). Similarly to the above one then defines the multimultisemigroup $\mathcal{M S}(\mathscr{C})$ for a small and fully additive 2-category $\mathscr{C}$. This multimultisemigroup then contains complete information about the combinatorics of composition in $\mathscr{C}$, including all multiplicities in the direct sum decomposition of the composition of two 1-morphisms. The multisemigroup $\mathcal{S}(\mathscr{C})$ contains only a "shadow" of this information, saying only which direct summands appear in the direct sum decomposition of the composition of two 1-morphisms.

\section{Additive And abelian 2-Representations}

Let $\mathscr{C}$ be a fiat 2-category. In $[\mathrm{MM}]$ we worked with abelian 2-representations of $\mathscr{C}$. In the present paper we would like to slightly change the approach and start with additive 2-representations (as was originally suggested in [Ro2]). We also formulate a direct 2-functorial link between these two classes of representations. 
4.1. Principal additive 2-representations. For $i \in \mathscr{C}$ let $\mathbb{P}_{i}: \mathscr{C} \rightarrow \mathfrak{A}_{\mathbb{k}}$ denote the $i$-th principal additive 2 -representation $\mathscr{C}\left(\mathbf{i},{ }_{-}\right)$. This 2 -representation assigns to each $j \in \mathscr{C}$ the category $\mathscr{C}(i, j)$, to each 1-morphism $F$ the functor given by the horizontal composition with $\mathrm{F}$, and to each 2-morphism $\alpha$ the natural transformation given by the horizontal composition with $\alpha$. This 2-representation is finitary as $\mathscr{C}$ is fiat.

Lemma 9 (Yoneda lemma). For any $\mathbf{M} \in \mathscr{C}$-amod we have an isomorphism

$$
\operatorname{Hom}_{C_{- \text {amod }}}\left(\mathbb{P}_{\mathbf{i}}, \mathbf{M}\right)=\mathbf{M}(\mathbf{i})
$$

Proof. Given $M \in \mathbf{M}(\mathbf{i})$ there is a unique homomorphism $\Phi_{M}: \mathbb{P}_{\mathbf{i}} \rightarrow \mathbf{M}$ such that $\Phi_{M}\left(\mathbb{1}_{\mathrm{i}}\right)=M$, namely the one sending $\mathrm{F} \in \mathscr{C}(\mathrm{i}, \mathrm{j})$ to $\mathrm{F} M$ and $\alpha: \mathrm{F} \rightarrow \mathrm{F}^{\prime}$ to $\alpha_{M}$.

Given $M, N \in \mathbf{M}(\mathrm{i})$ and $\tau \in \operatorname{Hom}_{\mathbf{M}(\mathrm{i})}(M, N)$, mapping $\mathrm{F} \in \mathscr{C}(\mathrm{i}, \mathrm{j})$ to $\mathrm{F}(\tau)$ defines a modification from $\Phi_{M}$ to $\Phi_{N}$. On the other hand, given a modification $\xi: \Phi_{M} \rightarrow \Phi_{N}$, let $\tau=\xi\left(\mathbb{1}_{i}\right)$. Then for any $\mathrm{F} \in \mathscr{C}(\mathbf{i}, \mathrm{j})$ the axioms of modification imply

$$
\mathrm{F}(\tau)=\mathrm{F}\left(\xi\left(\mathbb{1}_{i}\right)\right)=\xi\left(\mathrm{F} \mathbb{1}_{i}\right)=\xi(\mathrm{F})
$$

and hence $\xi$ is uniquely determined by $\tau$. The claim follows.

4.2. The abelianization 2-functor. Given $\mathbf{M} \in \mathscr{C}$-afmod we define a new abelian 2-representation $\overline{\mathbf{M}}$ of $\mathscr{C}$ in the following way. For $i \in \mathscr{C}$ define the category $\overline{\mathbf{M}}(\mathbf{i})$ as follows (cf. e.g. [Fr]): objects of $\overline{\mathbf{M}}(\mathrm{i})$ are all diagrams of the form $X \stackrel{\alpha}{\longrightarrow} Y$, where $X, Y \in \mathbf{M}(\mathrm{i})$ and $\alpha \in \operatorname{Hom}_{\mathbf{M}(\mathrm{i})}(X, Y)$. The homomorphism set

$$
\operatorname{Hom}_{\overline{\mathbf{M}}(\mathrm{i})}\left(X \stackrel{\alpha}{\longrightarrow} Y, X^{\prime} \stackrel{\alpha^{\prime}}{\longrightarrow} Y^{\prime}\right)
$$

is defined as the quotient of the linear space generated by all commutative diagrams as shown in the solid part of the following picture:

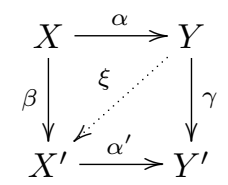

modulo the subspace spanned by all diagrams for which there exists $\xi$ as shown on the picture such that $\gamma=\alpha^{\prime} \xi$. The 2-action of $\mathscr{C}$ is defined on such diagrams in the natural way, that is component-wise, which makes $\overline{\mathbf{M}}$ into an abelian 2representation of $\mathscr{C}$. Extending 2-natural transformations and modifications in $\mathscr{C}$-afmod to diagrams component-wise defines a 2 -functor from $\mathscr{C}$-afmod to $\mathscr{C}$-mod which we will call the abelianization functor and denote by $\div: \mathscr{C}$-afmod $\rightarrow \mathscr{C}$-mod. Applying this 2-functor to $\mathbb{P}_{i}$ yields the principal abelian 2-representation $\overline{\mathbb{P}}_{i}$ considered in $[\mathrm{MM}]$.

Remark 10. Let $\mathfrak{T}_{\mathbb{k}}$ denote the locally small 2-subcategory of Cat with objects small triangulated $\mathbb{k}$-linear categories, 1-morphisms triangle functors and 2morphisms natural transformations of functors. Denote by $\mathscr{C}$-tmod the 2 -category of 2-representations of $\mathscr{C}$ in $\mathfrak{T}_{\mathbb{k}}$. Similarly to the above, using the usual construction of the homotopy category of an additive category, one defines a 2-functor from $\mathscr{C}$-afmod to $\mathscr{C}$-tmod. 
4.3. Additive subrepresentations of abelian representations. Assume that we are given $\mathbf{M} \in \mathscr{C}$-mod, $i \in \mathscr{C}$ and $X \in \mathbf{M}(\mathbf{i})$. For $\mathrm{j} \in \mathscr{C}$ define $\mathbf{M}_{X}(\mathrm{j})$ as $\operatorname{add}(\mathrm{F} X)$, where $\mathrm{F}$ runs through the set of all 1-morphisms in $\mathscr{C}(i, j)$. Since the number of 1-morphisms in $\mathscr{C}(i, j)$ is finite up to isomorphism, we have $\mathbf{M}_{X}(j) \in \mathfrak{A}_{\mathbb{k}}^{f}$. Via restriction from $\mathbf{M}, \mathbf{M}_{X}$ becomes a 2-representation of $\mathscr{C}$ and from the previous observation we obtain that $\mathbf{M}_{X} \in \mathscr{C}$-afmod. This is a very general and powerful tool for constructing new 2-representations.

4.4. Cell 2-representation. In this subsection we recall the construction of a special class of 2 -representations defined and studied in $[\mathrm{MM}]$. Let $\mathcal{L}$ be a (nonzero) left cell of $\mathcal{S}(\mathscr{C})$. To simplify notation, we will from now on identify indecomposable 1-morphisms in $\mathscr{C}$ with the corresponding classes in $\mathcal{S}(\mathscr{C})$, in particular, we will write $\mathrm{F} \in \mathcal{S}(\mathscr{C})$ instead of $[\mathrm{F}] \in \mathcal{S}(\mathscr{C})$.

There is $i=i_{\mathcal{L}} \in \mathscr{C}$ such that for any 1 -morphism $\mathrm{F} \in \mathcal{L}$ we have $\mathrm{F} \in \mathscr{C}(i, j)$ for some $j \in \mathscr{C}$. Consider $\overline{\mathbb{P}}_{i}$. For an indecomposable 1-morphism $F$ in some $\mathscr{C}(i, j)$ denote by $P_{\mathrm{F}}$ the indecomposable projective module $0 \rightarrow \mathrm{F}$ in $\overline{\mathbb{P}}_{\mathrm{i}}(\mathrm{j})$ and by $L_{\mathrm{F}}$ the unique simple top of $P_{\mathrm{F}}$. By [MM, Proposition 17], there exists the unique $\mathrm{G}_{\mathcal{L}} \in \mathcal{L}$ (called the Duflo involution in $\mathcal{L}$ ) such that the indecomposable projective module $P_{\mathbb{1}_{i}}$ has a unique quotient $N$ such that the simple socle of $N$ is isomorphic to $L_{\mathrm{G}_{\mathcal{L}}}$ and $\mathrm{F} N / L_{\mathrm{G}_{\mathcal{L}}}=0$ for any $\mathrm{F} \in \mathcal{L}$. Set $Q:=\mathrm{G}_{\mathcal{L}} L_{\mathrm{G}_{\mathcal{L}}}$. Then the additive 2-representation $\mathbf{C}_{\mathcal{L}}:=\left(\overline{\mathbb{P}}_{\mathrm{i}}\right)_{Q}$ is called the additive cell 2-representation of $\mathscr{C}$ associated to $\mathcal{L}$. The abelianization $\overline{\mathbf{C}}_{\mathcal{L}}$ of $\mathbf{C}_{\mathcal{L}}$ is called the abelian cell 2-representation of $\mathscr{C}$ associated to $\mathcal{L}$.

\section{Equivalent 2-Representations}

5.1. Abelian representations are defined by the action on projectives. Let $\mathbf{M} \in \mathscr{C}$-mod. As $\mathscr{C}$ is fiat, the action of any 1-morphism $\mathrm{F}$ in $\mathscr{C}$ on $\mathbf{M}$ is given by a functor biadjoint to an exact functor (representing the action of $\mathrm{F}^{*}$ ). In particular, it follows that any such $\mathrm{F}$ sends projective objects to projective objects. For every $i$ denote by $\mathbf{M}_{p r}(\mathbf{i})$ the additive subcategory of $\mathbf{M}(\mathbf{i})$ consisting of all projective objects. Then the restriction of the action of $\mathscr{C}$ defines the additive 2-representation $\mathbf{M}_{p r} \in \mathscr{C}$-afmod. The main result of this subsection is the following:

Theorem 11. The 2-representations $\mathbf{M}$ and $\overline{\mathbf{M}_{p r}}$ are equivalent.

Proof. We prove the claim by constructing a sequence of elementary equivalent 2-representations of $\mathscr{C}$ connecting $\mathbf{M}$ with $\overline{\mathbf{M}_{p r}}$.

Define the 2-representation $\mathbf{N}$ of $\mathscr{C}$ as follows: for $i \in \mathscr{C}$ the category $\mathbf{N}(i)$ has objects $(F X)_{(F)}$, where $X \in \mathbf{M}(j)$ and $F$ is a 1-morphism in $\mathscr{C}(j, i)$ for some $j$. For $(\mathrm{F} X)_{(\mathrm{F})},\left(\mathrm{F}^{\prime} X^{\prime}\right)_{\left(\mathrm{F}^{\prime}\right)} \in \mathbf{N}(\mathrm{i})$ set

$$
\operatorname{Hom}_{\mathbf{N}(\mathrm{i})}\left((\mathrm{F} X)_{(\mathrm{F})},\left(\mathrm{F}^{\prime} X^{\prime}\right)_{\left(\mathrm{F}^{\prime}\right)}\right):=\operatorname{Hom}_{\mathbf{M}(\mathrm{i})}\left(\mathrm{F} X, \mathrm{~F}^{\prime} X^{\prime}\right) .
$$

Define the action of 1-morphisms of $\mathscr{C}$ on objects by $\mathrm{G}(\mathrm{F} X)_{(\mathrm{F})}:=(\mathrm{G} \circ \mathrm{F} X)_{(\mathrm{GoF})}$. Define the action of 1-morphisms of $\mathscr{C}$ on morphisms by inducing the action from the one on $\mathbf{M}(\mathbf{i})$ in the natural way. Define the action of 2-morphisms similarly by inducing it from $\mathbf{M}(i)$ in the natural way. This gives an abelian 2-representation of $\mathscr{C}$. Sending $(\mathrm{F} X)_{(\mathrm{F})} \in \mathbf{N}(\mathrm{i})$ to $\mathrm{F} X \in \mathbf{M}(\mathrm{i})$ and using the identity map on morphisms we get the forgetful 2-natural transformation from $\mathbf{N}$ to $\mathbf{M}$ which obviously restricts to an equivalence for every i. Hence $\mathbf{M}$ and $\mathbf{N}$ are elementary equivalent. 
For any $i \in \mathscr{C}$ and any object $X \in \mathbf{M}(\mathbf{i})$ fix some projective presentation $P_{X} \stackrel{\alpha}{\rightarrow}$ $Q_{X} \rightarrow X$ of $X$ in $\mathbf{M}(\mathrm{i})$. The assignment

$$
(\mathrm{F} X)_{(\mathrm{F})} \mapsto\left(\mathrm{F} P_{X} \stackrel{\mathrm{F} \alpha}{\longrightarrow} \mathrm{F} Q_{X}\right)
$$

extends to a 2-natural transformation $\mathbf{N} \rightarrow \overline{\mathbf{M}_{p r}}$ in the obvious way. Clearly, the restriction of this 2-natural transformation to any $i$ is an equivalence. Hence $\mathbf{N}$ and $\overline{\mathbf{M}_{p r}}$ are elementary equivalent. The claim of the theorem follows.

5.2. Comparison of cell 2-representations. In this subsection we present the additive adaptation of [MM, Theorem 1]. First we note that our definition of an abelian cell 2-representation is slightly different from the one in [MM] (here we define the abelian cell 2-representation as abelianization of a certain additive 2subrepresentation $\mathbf{N}$ of $\overline{\mathbb{P}}_{\mathbf{i}}$, while in $[\mathrm{MM}]$ the abelian cell 2-representation was defined as an abelian 2-subrepresentation $\mathbf{M}$ of $\overline{\mathbb{P}}_{\mathbf{i}}$ such that $\mathbf{N}=\mathbf{M}_{p r}$ ). By Theorem 11, these two different definitions produce equivalent 2-representations. We also note that we slightly change the notation from $[\mathrm{MM}]$ because of the introduction of the abelianization functor (and thus $\mathbf{C}_{\mathcal{L}}$ is an additive 2-representation in this paper while it was abelian in $[\mathrm{MM}])$. We think our present notation is more natural.

Let $\mathscr{C}$ be a fiat 2-category, $\mathcal{J}$ a two-sided cell of $\mathscr{C}$ and $\mathcal{L}$ a left cell in $\mathcal{J}$. The cell $\mathcal{J}$ is called strongly regular (see [MM, 4.8]) provided that any two different left cells in $\mathcal{J}$ are not comparable with respect to $\leq_{L}$, and the intersection of each left and each right cell in $\mathcal{J}$ consists of exactly one element. For example, all two-sided cells for the fiat 2-category $\mathscr{C}_{A}$ in Example 2 are strongly regular. Similarly, all two-sided cells for the fiat 2-category $\mathscr{S}_{\mathfrak{s l}_{n}}$ in Example 3 are strongly regular (however, this is not the case if $\mathfrak{g}$ is not of type $A$ ). Assume that $\mathcal{J}$ is strongly regular. Then for any $\mathrm{F} \in \mathcal{J}$ the intersection of the left cell of $\mathrm{F}$ with the right cell of $\mathrm{F}^{*}$ consists of a unique element, say $\mathrm{H}$. Let $m_{\mathrm{F}}$ denote the multiplicity of $\mathrm{H}$ in $\mathrm{F}^{*} \circ \mathrm{F}$.

Proposition 12. Assume that $\mathcal{J}$ is strongly regular and that

$$
\text { the function } \mathrm{F} \mapsto m_{\mathrm{F}} \text { is constant on right cells of } \mathcal{J} \text {. }
$$

Then for any two left cells $\mathcal{L}$ and $\mathcal{L}^{\prime}$ in $\mathcal{J}$ the 2-representations $\mathbf{C}_{\mathcal{L}}$ and $\mathbf{C}_{\mathcal{L}^{\prime}}$ are equivalent and, similarly, their abelianizations are equivalent as well.

Note that in $[\mathrm{MM}]$ it was shown that the technical condition of Proposition 12 is satisfied for strongly regular cells in Examples 2 and 3.

Proof. Let $\mathrm{H}$ be the unique element in the intersection of $\mathcal{L}^{\prime}$ with the right cell of $\mathrm{G}_{\mathcal{L}}$. Assume $\mathrm{G}_{\mathcal{L}} \in \mathscr{C}(\mathrm{i}, \mathrm{i})$ and $\mathrm{G}_{\mathcal{L}^{\prime}} \in \mathscr{C}(\mathrm{j}, \mathrm{j})$. By Lemma 9 we have a unique homomorphism $\Phi: \mathbb{P}_{i} \rightarrow \overline{\mathbb{P}}_{j}$ mapping $\mathbb{1}_{i}$ to $L_{\mathrm{H}}$.

Let $\mathbf{N}$ denote the additive 2-subrepresentation of $\mathbb{P}_{i}$ obtained by restriction to the full additive subcategory generated by all 1-morphisms $\mathrm{F}$ satisfying $\mathrm{F} \geq_{L} \mathrm{G}_{\mathcal{L}}$. Let $\mathbf{M}$ denote the additive 2-subrepresentation of $\overline{\mathbb{P}}_{j}$ obtained by restriction to the full additive subcategory generated by $\mathrm{F} L_{\mathrm{H}}$ for all 1-morphisms $\mathrm{F}$ satisfying $\mathrm{F} \geq_{L} \mathrm{G}_{\mathcal{L}}$. From [MM, Theorem 43(a)] it follows that the latter additive subcategory coincides with the additive closure of $\mathrm{F} L_{\mathrm{H}}, \mathrm{F} \in \mathcal{L}^{\prime}$, and hence with $\mathbf{C}_{\mathcal{L}^{\prime}}$. Thus $\Phi$ induces, by restriction, a homomorphism $\Phi^{\prime}: \mathbf{N} \rightarrow \mathbf{C}_{\mathcal{L}^{\prime}}$.

Let $\mathrm{F} \stackrel{\alpha}{\longrightarrow} \mathrm{G}_{\mathcal{L}}$ be the object of $\overline{\mathbf{N}}(\mathrm{i})$ obtained from $L_{\mathrm{G}_{\mathcal{L}}}$ by deleting all summands outside $\mathbf{N}(\mathrm{i})$. From [MM, Theorem 43(a)] it follows that the abelianization of $\Phi^{\prime}$ sends $\mathrm{F} \stackrel{\alpha}{\longrightarrow} \mathrm{G}_{\mathcal{L}}$ to a simple object in $\overline{\mathbf{C}}_{\mathcal{L}^{\prime}}$ (i) isomorphic to the top of the 
indecomposable projective object $0 \rightarrow \mathrm{G}_{\mathcal{L}} L_{\mathrm{H}}$. This induces an equivalence between $\mathbf{C}_{\mathcal{L}}$ and $\left(\overline{\mathbf{C}}_{\mathcal{L}^{\prime}}\right)_{p r}$. Mapping $P$ to $0 \rightarrow P$ for any object $P$ induces an obvious equivalence from $\mathbf{C}_{\mathcal{L}^{\prime}}$ to $\left(\overline{\mathbf{C}}_{\mathcal{L}^{\prime}}\right)_{p r}$ and the additive claim follows. The abelian claim follows from Theorem 11 applying the abelianization functor.

\section{Annihilators of CELl 2-REPRESEntations}

6.1. 2-ideals and quotients. A 2-ideal $\mathscr{I}$ of $\mathscr{C}$ consists of the following data:

- the same objects as $\mathscr{C}$;

- the same 1-morphisms as $\mathscr{C}$;

- for any $i, j \in \mathscr{C}$ and any 1-morphisms $F, G \in \mathscr{C}(i, j)$ a $\mathbb{k}$-subspace $\operatorname{Hom}_{\mathscr{I}}(\mathrm{F}, \mathrm{G}) \subset \operatorname{Hom}_{\mathscr{C}(\mathrm{i}, \mathrm{j})}(\mathrm{F}, \mathrm{G})$ of 2-morphisms;

such that for any 2-morphisms $\xi$ in $\mathscr{I}$ and $\alpha, \beta$ in $\mathscr{C}$ the compositions $\alpha \circ_{0} \xi \circ_{0} \beta$ and $\alpha \circ_{1} \xi \circ_{1} \beta$ are in $\mathscr{I}$ whenever the expression makes sense.

If $\mathscr{I}$ is a 2 -ideal of $\mathscr{C}$, then we can define the quotient 2-category $\mathscr{C} / \mathscr{I}$ as follows: $\mathscr{C} / \mathscr{I}$ has the same objects and 1-morphisms as $\mathscr{C}$, and for for any i, j $\in \mathscr{C}$ and any 1-morphisms F, $\mathrm{G} \in \mathscr{C}(\mathrm{i}, \mathrm{j})$ we set

$$
\operatorname{Hom}_{\mathscr{C} / \mathscr{I}}(\mathrm{F}, \mathrm{G}):=\operatorname{Hom}_{\mathscr{C}(\mathrm{i}, \mathrm{j})}(\mathrm{F}, \mathrm{G}) / \operatorname{Hom}_{\mathscr{I}}(\mathrm{F}, \mathrm{G}) ;
$$

all compositions in $\mathscr{C} / \mathscr{I}$ are induced by the corresponding compositions in $\mathscr{C}$. The fact that this is well-defined follows directly from the axioms of a 2-ideal.

If $\Phi: \mathscr{C} \rightarrow \mathscr{A}$ is a 2-functor between two fiat categories, define the kernel $\operatorname{Ker}(\Phi)$ as the datum consisting of the same objects and 1-morphisms as $\mathscr{C}$, and for any $i, j \in \mathscr{C}$ and any 1 -morphisms $F, G \in \mathscr{C}(i, j)$ the subspace $\operatorname{Hom}_{\operatorname{Ker}(\Phi)}(F, G)$ which coincides with the kernel of the $\mathbb{k}$-linear map

$$
\Phi_{\mathrm{F}, \mathrm{G}}: \operatorname{Hom}_{\mathscr{C}_{(i, j)}}(\mathrm{F}, \mathrm{G}) \rightarrow \operatorname{Hom}_{\mathscr{A}(\Phi(\mathrm{i}), \Phi(\mathrm{j}))}(\Phi(\mathrm{F}), \Phi(\mathrm{G}))
$$

given by the application of $\Phi$. We have the following usual property that 2-ideals are exactly kernels of 2-functors.

Lemma 13. (a) $\operatorname{Ker}(\Phi)$ is a 2-ideal of $\mathscr{C}$.

(b) For any 2-ideal $\mathscr{I}$ of $\mathscr{C}$ there is a 2-category $\mathscr{A}$ and a 2-functor $\Phi: \mathscr{C} \rightarrow \mathscr{A}$ such that $\operatorname{Ker}(\Phi)=\mathscr{I}$.

Proof. Claim (a) is checked by a direct computation. To prove claim (b) just take $\mathscr{A}=\mathscr{C} / \mathscr{I}$ and let $\Phi$ be the natural projection.

Lemma 14. Let $\mathscr{C}$ be a fiat 2-category and $\mathscr{I}$ a 2-ideal.

(i) The image in $\mathscr{C} / \mathscr{I}$ of an indecomposable 1-morphism in $\mathscr{C}$ is either indecomposable or zero.

(ii) Every left, right or 2-sided cell of $\mathscr{C}$ descends either to a left, right or 2-sided cell of $\mathscr{C} / \mathscr{I}$, respectively, or to zero.

Proof. Claim (i) follows from definitions and the fact that the quotient of a local endomorphism algebra of an indecomposable 1-morphism is either local or zero.

For two indecomposable 1-morphisms $\mathrm{F}$ and $\mathrm{G}$ we have $\mathrm{F} \leq_{L} \mathrm{G}$ provided that there exists $\mathrm{H}$ such that $\mathrm{G}$ is isomorphic to a direct summand of $\mathrm{H} \circ \mathrm{F}$. Let $\Phi: \mathscr{C} \rightarrow \mathscr{C} / \mathscr{I}$ 
be the quotient functor, then $\Phi(\mathrm{G})$ is either zero or isomorphic to a direct summand of $\Phi(\mathrm{H}) \circ \Phi(\mathrm{F})$. Hence $\Phi(\mathrm{F}) \leq_{L} \Phi(\mathrm{G})$ if $\Phi(\mathrm{G})$ is nonzero and claim (ii) follows in the case of left cells. The other cases are similar.

6.2. $\mathcal{J}$-simple fiat categories. Let $\mathscr{C}$ be a fiat 2 -category and $\mathcal{J}$ a non-zero twosided cell in $\mathscr{C}$. We will say that $\mathscr{C}$ is $\mathcal{J}$-simple provided that for every non-trivial 2-ideal $\mathscr{I}$ in $\mathscr{C}$ there exists $\mathrm{F} \in \mathcal{J}$ such that $\mathscr{I}$ contains $\operatorname{id}_{\mathrm{F}}$. This is the closest analogue to the notion of a simple 2-category which makes sense in our context. Our main result of this subsection is the following:

Theorem 15. Let $\mathscr{C}$ be a fiat 2-category and $\mathcal{J}$ a non-zero two-sided cell in $\mathscr{C}$. Then there is a unique 2 -ideal $\mathscr{I}$ in $\mathscr{C}$ such that $\mathscr{C} / \mathscr{I}$ is $\mathcal{J}$-simple.

To prove this theorem we will need the following lemmata.

Lemma 16. (i) If $\mathscr{I}$ is a 2 -ideal of $\mathscr{C}$ containing $\mathrm{id}_{\mathrm{F}}$ for some $\mathrm{F} \in \mathcal{J}$, then $\mathscr{I}$ contains $\operatorname{id}_{\mathrm{G}}$ for any $\mathrm{G} \geq_{J} \mathrm{~F}$.

(ii) Assume that $\mathscr{I}$ is a 2-ideal of $\mathscr{C}$ generated by $\mathrm{id}_{\mathrm{F}}$ for some indecomposable F. For any 1-morphisms $\mathrm{G}$ and $\mathrm{H}$ let $\operatorname{Hom} \mathscr{G}(\mathrm{G}, \mathrm{H})$ denote the subspace of $\operatorname{Hom}_{\mathscr{C}}(\mathrm{G}, \mathrm{H})$ generated by all morphisms which factor through some $\mathrm{K}$ such that $\mathrm{K} \geq_{J} \mathrm{~F}$. Then $\mathscr{J}$ is a 2 -ideal of $\mathscr{C}$ and $\mathscr{I}=\mathscr{J}$.

Proof. As $\mathscr{I}$ is a 2-ideal containing $\mathrm{id}_{\mathrm{F}}$, it also contains $\mathrm{id}_{\mathrm{G}}$ for any $\mathrm{G}=\mathrm{H} \circ \mathrm{F} \circ \mathrm{H}^{\prime}$ (using the horizontal composition of $\mathrm{id}_{\mathrm{F}}$ with $\mathrm{id}_{\mathrm{H}}$ on the left and $\mathrm{id}_{\mathrm{H}^{\prime}}$ on the right). Composing $\mathrm{id}_{\mathrm{G}}$ with projections onto all direct summand of $\mathrm{G}$ we get that $\mathscr{I}$ also contains $\mathrm{id}_{\mathrm{G}^{\prime}}$ for any direct summand $\mathrm{G}^{\prime}$ of $\mathrm{G}$. Claim (i) now follows from the definition of $\geq_{J}$.

That $\mathscr{J}$ is a 2 -ideal of $\mathscr{C}$ follows directly from the fact that the additive closure of all $\mathrm{K}$ such that $\mathrm{K} \geq_{J} \mathrm{~F}$ is closed with respect to horizontal composition. As $\mathrm{id}_{\mathrm{F}}$ is contained in $\mathscr{J}$, we have $\mathscr{I} \subset \mathscr{J}$. On the other hand, from claim (i) we know that $\mathscr{I}$ contains $\mathrm{id}_{\mathrm{K}}$ for any $\mathrm{K} \geq_{J} \mathrm{~F}$. Hence $\mathscr{J} \subset \mathscr{I}$ implying claim (ii).

Remark 17. A 2-ideal $\mathscr{I}$ of $\mathscr{C}$ is called thick if it is generated by $\mathrm{id}_{\mathrm{F}_{i}}$ for some collection $\left\{\mathrm{F}_{i}\right\}$ of 1-morphisms in $\mathscr{C}$ (these kinds of ideals, under the name of "tensor ideals" were considered, for example, in [Os]). From Lemma 16(ii) it follows that thick 2-ideals of $\mathscr{C}$ are in a natural bijection with anti-chains of the partially ordered set of all two-sided cells of $\mathscr{C}$ with respect to the partial order $\leq_{J}$.

Lemma 18. The claim of Theorem 15 is true under the assumption that $\mathcal{J}$ is the unique maximal non-zero two-sided cell of $\mathscr{C}$ (with respect to $\leq_{J}$ ).

Proof. Let $\mathscr{J}$ be a 2-ideal of $\mathscr{C}$ that does not contain $\operatorname{id}_{\mathrm{F}}$ for any $\mathrm{F} \in \mathcal{J}$. Then for any $\mathrm{F} \in \mathcal{J}$ we have the ideal $\operatorname{Hom} \mathscr{J}(\mathrm{F}, \mathrm{F})$ of the finite-dimensional $\mathbb{k}$-algebra $\operatorname{Hom}_{\mathscr{C}(i, j)}(F, F)$ which does not coincide with the latter algebra. Hence Hom $\mathscr{J}(F, F)$ is contained in the Jacobson radical of $\operatorname{Hom}_{\mathscr{C}(i, j)}(F, F)$. Note that the Jacobson radical is stable under taking sums of subideals.

Let $\mathscr{I}$ denote the sum of all 2-ideals of $\mathscr{C}$ that do not contain any id $\mathrm{id}_{\mathrm{F}}$ for $\mathrm{F} \in \mathcal{J}$. From the previous paragraph it follows that for $\mathrm{F} \in \mathcal{J}$ the ideal $\operatorname{Hom}_{\mathscr{I}}(\mathrm{F}, \mathrm{F})$ of $\operatorname{Hom}_{\mathscr{C}_{(i, j)}}(\mathrm{F}, \mathrm{F})$ is contained in the Jacobson radical of $\operatorname{Hom}_{\mathscr{C}_{(i, j)}}(\mathrm{F}, \mathrm{F})$. In particular, $\operatorname{Hom}_{\mathscr{I}}(\mathrm{F}, \mathrm{F})$ does not contain $\mathrm{id}_{\mathrm{F}}$. We will now show that $\mathscr{I}$ is the unique 2 -ideal such that $\mathscr{C} / \mathscr{I}$ is $\mathcal{J}$-simple. 
Indeed, consider the quotient 2-category $\mathscr{C} / \mathscr{I}$. Let $\mathscr{I}^{\prime}$ be a 2-ideal of $\mathscr{C} / \mathscr{I}$ which does not contain any $\mathrm{id}_{\mathrm{F}}$ for $\mathrm{F} \in \mathcal{J}$. Then the full preimage of $\mathscr{I}^{\prime}$ in $\mathscr{C}$ also has this property and hence is contained in $\mathscr{I}$ by the construction of $\mathscr{I}$. Hence $\mathscr{I}^{\prime}=\mathscr{I}$ and $\mathscr{C} / \mathscr{I}$ is $\mathcal{J}$-simple.

For uniqueness, let $\mathscr{I}^{\prime}$ be another 2 -ideal of $\mathscr{C}$ such that $\mathscr{C} / \mathscr{I}^{\prime}$ is $\mathcal{J}$-simple. Then Lemma 16(i) implies that $\mathscr{I}^{\prime}$ does not contain any id $\mathrm{F}$ for $\mathrm{F} \in \mathcal{J}$ and hence $\mathscr{I}^{\prime} \subset \mathscr{I}$. The image of $\mathscr{I}$ in $\mathscr{C} / \mathscr{I}^{\prime}$ is a 2 -ideal which does not contain any $\mathrm{id}_{\mathrm{F}}$ for $\mathrm{F} \in \mathcal{J}$. As $\mathscr{C} / \mathscr{I}^{\prime}$ is $\mathcal{J}$-simple, we thus get $\mathscr{I}^{\prime}=\mathscr{I}$. The claim follows.

Proof of Theorem 15. Denote by $\mathscr{J}$ the 2 -ideal of $\mathscr{C}$ generated by $\mathrm{id}_{\mathrm{G}}$ for all 1 morphisms $\mathrm{G}$ in $\mathscr{C}$ such that $\mathrm{F} \nsupseteq_{J} \mathrm{G}$ for any $\mathrm{F} \in \mathcal{J}$. If $\mathscr{I}$ is a 2 -ideal of $\mathscr{C}$ which does not contain any $\mathrm{id}_{\mathrm{F}}$ for $\mathrm{F} \in \mathcal{J}$, then the image of $\mathscr{J}$ in $\mathscr{C} / \mathscr{I}$ does not contain any $\mathrm{id}_{\mathrm{F}}$ for $\mathrm{F} \in \mathcal{J}$ either. Hence, if $\mathscr{C} / \mathscr{I}$ is $\mathcal{J}$-simple, then $\mathscr{J} \subset \mathscr{I}$. This means that without loss of generality we may assume (by going to $\mathscr{C} / \mathscr{J}$ ) that $\mathscr{J}$ is trivial. However, in the latter case the definition of $\mathscr{J}$ implies that $\mathcal{J}$ is the unique maximal two-sided cell of $\mathscr{C}$. Now the claim follows from Lemma 18.

6.3. Annihilators of cell 2-representations. In this section we describe annihilators of cell 2-representations. The following theorem generalizes [Ag, Lemma 3.25].

Theorem 19. Let $\mathcal{J}$ be a two-sided cell in $\mathscr{C}$ and $\mathcal{L}$ be a left cell in $\mathcal{J}$. Then the "image" 2-category $\mathscr{C} / \operatorname{Ker}\left(\mathbf{C}_{\mathcal{L}}\right)$ of the cell 2-representation $\mathbf{C}_{\mathcal{L}}$ is $\mathcal{J}$-simple.

Proof. Let $\mathscr{I}$ be the 2-ideal of $\mathscr{C}$ given by Theorem 15 . Consider the quotient 2-category $\mathscr{A}:=\mathscr{C} / \mathscr{I}$. Then $\mathcal{J}$ descends, by Lemma 14(ii), to a two-sided cell for $\mathscr{A}$ which we will denote by $\mathcal{J}_{\mathscr{A}}$. Similarly, we have the left cell $\mathcal{L}_{\mathscr{A}}$ of $\mathscr{A}$ coming from $\mathcal{L}$. The cell 2-representation $\mathbf{C}_{\mathcal{L}}$ of $\mathscr{A}$ becomes a 2-representation of $\mathscr{C}$ by composing the 2-functor $\mathbf{C}_{\mathcal{L} \mathscr{A}}$ with the quotient 2-functor $\mathscr{C} \rightarrow \mathscr{A}$, so by definition $\mathscr{I} \subset \operatorname{Ker}\left(\mathbf{C}_{\mathcal{L}_{\mathscr{A}}}\right)$. On the other hand $\mathscr{A}$ is $\mathcal{J}$-simple, so no non-trivial ideal of $\mathscr{A}$ can annihilate $\mathbf{C}_{\mathcal{L}_{\mathscr{A}}}$ showing $\operatorname{Ker}\left(\mathbf{C}_{\mathcal{L}_{\mathscr{A}}}\right) \subset \mathscr{I}$. This yields $\operatorname{Ker}\left(\mathbf{C}_{\mathcal{L}_{\mathscr{A}}}\right)=\mathscr{I}$.

Let $i \in \mathscr{C}$ be such that $\mathrm{G}_{\mathcal{L}} \in \mathscr{C}(\dot{i}, \mathbf{i})$. Similarly to the proof of Proposition 12 , the unique homomorphism from $\mathbb{P}_{i}$ to $\mathbf{C}_{\mathcal{L}_{\mathscr{A}}}$ sending $\mathbb{1}_{i}$ to $L_{\mathrm{G}_{\mathcal{L}}}$ gives rise to a homomorphism $\Phi: \mathbf{C}_{\mathcal{L}} \rightarrow\left(\overline{\mathbf{C}}_{\mathcal{L}_{\mathscr{A}}}\right)_{p r}$. The abelianization $\bar{\Phi}$ sends the simple top $L$ of $0 \rightarrow \mathrm{G}_{\mathcal{L}} L_{\mathrm{G}_{\mathcal{L}}}$ to the corresponding simple object $L^{\prime}$ in $\overline{\left(\overline{\mathbf{C}}_{\mathcal{L}_{\mathscr{A}}}\right)_{p r}}(\mathrm{i})$.

From [MM, Proposition 17(b)] it follows that indecomposable projectives for $\overline{\mathbf{C}}_{\mathcal{L}}$ and $\overline{\mathbf{C}}_{\mathcal{L}_{\mathscr{A}}}$ have the form $\mathrm{F} L$ and $\mathrm{F} L^{\prime}$ for $\mathrm{F} \in \mathcal{L}$ or $\mathrm{F} \in \mathcal{L}_{\mathscr{A}}$, respectively. This means that $\Phi$ sends indecomposable objects to indecomposable objects and, moreover, [MM, Lemma 21] also implies that $\Phi$ is full.

Furthermore, using adjunction, for $\mathrm{F}, \mathrm{G} \in \mathcal{J}$ we have

$$
\operatorname{Hom}(\mathrm{F} L, \mathrm{G} L) \cong \operatorname{Hom}\left(L, \mathrm{~F}^{*} \circ \mathrm{G} L\right) .
$$

By [MM, Lemma 19], the dimension of the latter space equals the multiplicity of $\mathrm{G}_{\mathcal{L}}$ in $\mathrm{F}^{*} \circ \mathrm{G}$. Similarly for $\mathscr{A}$. As the decomposition multiplicities for $\mathscr{C}$ and $\mathscr{A}$ coincide, it follows that $\Phi$ is an equivalence when restricted to any $j$. This implies that $\operatorname{Ker}\left(\mathbf{C}_{\mathcal{L}}\right)=\operatorname{Ker}\left(\mathbf{C}_{\mathcal{L}_{\mathscr{A}}}\right)$ which yields $\operatorname{Ker}\left(\mathbf{C}_{\mathcal{L}}\right)=\mathscr{I}$. The claim follows.

We end this subsection with an example of a cell 2-representation, which is not strongly simple, but whose "image" 2-category is simple in the above sense. 
Example 20. Consider [MM, Example 7.1] in more detail for Dynkin type $B_{2}$. Keeping the notation from this example, we let $\mathscr{S}$ denote the (strict) 2-category defined as follows: it has one object $i$, which we identify with (a small category equivalent to) the regular block $\mathcal{O}_{0}$ of the BGG category $\mathcal{O}$ in type $B_{2}$; its 1 morphisms are projective functors $\theta$ on $\mathcal{O}_{0}$; and its 2-morphisms are natural transformations of functors. Indecomposable projective functors are indexed by elements of the Weyl group $W$ of type $B_{2}$, which has elements $\{e, s, t, s t, t s, s t s, t s t, s t s t\}$. Cells are again given by Kahzdan-Lusztig combinatorics, the two-sided cells are $\mathcal{J}_{e}=\{e\}, \mathcal{J}_{s, t}=\{s, t, s t, t s, s t s, t s t\}$ and $\mathcal{J}_{\text {stst }}=\{s t s t\}$. The middle cell splits into two left cells $\mathcal{L}_{1}=\{s, s t, s t s\}$ and $\mathcal{L}_{2}=\{t, t s, t s t\}$ (note that our left cells are Kazhdan-Lusztig's right cells and vice versa). Right cells are given by $\mathcal{R}_{1}=\{s, t s, s t s\}$ and $\mathcal{R}_{2}=\{t, s t, t s t\}$, so this is not strongly regular. Indeed in the cell 2-representation $\overline{\mathbf{C}}_{\mathcal{L}_{1}}$, which is generated by $L_{s}$ for the Duflo involution $s$, the simple module $L_{s t}$ is annihilated by $\theta_{s}$ (and hence by $\theta_{s t s}$ as well), and is mapped to the projective object indexed by $s t$ in $\overline{\mathbf{C}}_{\mathcal{L}_{1}}$ by $\theta_{t}$. Applying $\theta_{s}$ to this projective object, we obtain the direct sum of the two projectives in $\overline{\mathbf{C}}_{\mathcal{L}_{1}}$ labeled by $s$ and sts. One can check by explicit computations that it is impossible to split this direct sum using only morphisms in the image of the action of $\mathscr{S}$. As the functor $\theta_{s} \theta_{t}$ is indecomposable, this implies that $\overline{\mathbf{C}}_{\mathcal{L}_{1}}$ is not strongly simple. On the other hand, Theorem 19 implies that $\mathscr{S} / \operatorname{Ker}\left(\mathbf{C}_{\mathcal{L}_{1}}\right)$ is $\mathcal{J}_{s, t}$-simple.

6.4. Left 2-ideals. Let $\mathscr{A}$ be a $\mathbb{k}$-linear 2-category. A left 2-ideal $\mathscr{I}$ of $\mathscr{A}$ consists of the same objects as $\mathscr{A}$, and for each $i, j \in \mathscr{I}$ an ideal $\mathscr{I}(i, j)$ in $\mathscr{A}(i, j)$ such that $\mathscr{I}$ is stable under left horizontal multiplication with both 1 - and 2-morphisms in $\mathscr{A}$. For example, we can view $\mathscr{A}\left(\mathrm{k},{ }_{-}\right)$as a left 2 -ideal $\mathscr{I}_{\mathrm{k}}:=\mathscr{I}_{\mathrm{k}}^{\mathscr{A}}$ in $\mathscr{A}$ in the following way:

$$
\mathscr{I}_{\mathrm{k}}(\mathrm{i}, \mathrm{j}):= \begin{cases}\mathscr{A}(\mathrm{k}, \mathrm{j}), & \mathrm{i}=\mathrm{k} ; \\ 0, & \text { else. }\end{cases}
$$

It is easy to see that $\mathscr{I}_{\mathrm{k}}$ is generated, as a left 2-ideal, by the 2 -morphism id $\mathbb{1}_{\mathrm{k}}$.

Let $\mathscr{C}$ be a fiat 2 -category. Given a left 2 -ideal $\mathscr{J}$ contained in $\mathscr{I}_{i}$, we can define the quotient 2-representation $\mathbb{P}_{i} / \mathscr{J}$ by letting

$$
\left(\mathbb{P}_{\mathrm{i}} / \mathscr{J}\right)(\mathrm{j}):=\mathbb{P}_{\mathrm{i}}(\mathrm{j}) / \mathscr{J}(\mathrm{i}, \mathrm{j})
$$

with the action of $\mathscr{C}$ induced from that on $\mathbb{P}_{i}$. It is easy to check that $\mathbb{P}_{i} / \mathscr{J}$ is finitary.

Left 2-ideals appear naturally as annihilators of objects for 2-representations. Let $\mathbf{M}$ be a 2-representation of $\mathscr{C}$ and $X \in \mathbf{M}(i)$. Define $\operatorname{Ann}_{\mathscr{C}}(X)$ as the set of all 2-morphisms $\alpha$ such that either $\alpha=0$ or $\mathbf{M}(\alpha)_{X}$ is defined and $\mathbf{M}(\alpha)_{X}=0$. Then it is easy to see that $\operatorname{Ann}_{\mathscr{C}}(X)$ has the natural structure of a left 2-ideal of $\mathscr{C}$. Note that $\operatorname{Ann}_{\mathscr{C}}(X) \subset \mathscr{I}_{\mathrm{i}}$ by definition.

Proposition 21. Assume that $\mathscr{C}$ has a unique maximal two-sided cell $\mathcal{J}$ and let $\mathcal{L}$ be a left cell in $\mathcal{J}$. Then for $\mathrm{i}:=\mathrm{i}_{\mathcal{L}}$ there is a unique maximal left 2 -ideal $\mathscr{I}$ of $\mathscr{C}$ contained in $\mathscr{I}_{\mathrm{i}}$ such that $\mathscr{I}$ does not contain $\mathrm{id}_{\mathrm{G}_{\mathcal{L}}}$.

Proof. Let $\mathscr{I} \subset \mathscr{I}_{\mathrm{i}}$ be a left 2 -ideal of $\mathscr{C}$ which does not contain id $\mathrm{G}_{\mathcal{L}}$. Similarly to the proof of Lemma 16 one shows that $\mathscr{I}$ does not contain any $\operatorname{id}_{\mathrm{F}}$ for $\mathrm{F} \in \mathcal{L}$. This implies that the part of $\mathscr{I}$ inside the local algebra $\operatorname{End}_{\mathscr{C}(i, j)}(F)$ is properly contained in the radical. This property is preserved if one consider the sum of all $\mathscr{I}$ 's. The claim follows. 
6.5. An alternative construction of cell modules. Let $\mathcal{J}$ be a two-sided cell in $\mathscr{C}$ and $\mathcal{L}$ a left cell in $\mathcal{J}$. Let $\mathscr{I}$ be the 2-ideal in $\mathscr{C}$ given by Theorem 15 (i.e. $\mathscr{A}:=\mathscr{C} / \mathscr{I}$ is $\mathcal{J}$-simple). For $i:=i_{\mathcal{L}}$ let $\mathscr{J}$ be the unique maximal left 2 -ideal of $\mathscr{A}$ contained in $\mathscr{I}_{i}^{\mathscr{A}}$ which does not contain $\mathrm{id}_{\mathrm{F}}$ for any $\mathrm{F} \in \mathcal{L}$. Then the $2-$ representation $\mathbb{P}_{i}^{\mathscr{A}} / \mathscr{J}$ of $\mathscr{A}$ has the natural structure of a 2 -representation of $\mathscr{C}$ via the quotient 2-functor $\mathscr{C} \rightarrow \mathscr{A}$. For $\mathrm{j} \in \mathscr{C}$ let $\mathbf{D}_{\mathcal{L}}(\mathrm{j})$ denote the full subcategory of $\left(\mathbb{P}_{i}^{\mathscr{A}} / \mathscr{J}\right)(\mathrm{j})$ containing all 1 -morphisms $\mathrm{F}$ from $\mathscr{A}(\mathrm{i}, \mathrm{j})$ corresponding to $\mathcal{L}$. Then $\mathbf{D}_{\mathcal{L}}$ inherits the structure of a 2-representation of $\mathscr{C}$ by restriction.

Proposition 22. The 2-representations $\mathbf{D}_{\mathcal{L}}$ and $\mathbf{C}_{\mathcal{L}}$ are elementary equivalent.

Proof. From Theorem 19 it follows that $\mathscr{I}$ annihilates $L_{\mathrm{G}_{\mathcal{L}}}$ in $\overline{\mathbb{P}}_{\mathrm{i}}$. Therefore, $\mathbf{C}_{\mathcal{L}}=$ $\left(\overline{\mathbb{P}}_{\mathrm{i}}\right)_{\mathrm{G}_{\mathcal{L}} L_{\mathrm{G}_{\mathcal{L}}}}$ is indeed a 2-representation of $\mathscr{A}$. Mapping $\mathbb{1}_{\mathrm{i}}$ to $L_{\mathrm{G}_{\mathcal{L}}}$ extends to a homomorphism $\Phi$ from $\mathbb{P}_{i}^{\mathscr{A}}$ to $\left(\overline{\mathbb{P}}_{i}\right)_{L_{\mathrm{G}_{\mathcal{L}}}}$ (the latter considered as a 2-representation of $\mathscr{A}$ ). Let $L$ be the simple head of the indecomposable projective object

$$
0 \rightarrow \mathrm{G}_{\mathcal{L}} L_{\mathrm{G}_{\mathcal{L}}} \in \overline{\left(\overline{\mathbb{P}}_{\mathrm{i}}\right)_{L_{\mathrm{G}_{\mathcal{L}}}}}(\mathrm{i}) .
$$

We obviously have $\mathscr{J}^{\prime}:=\operatorname{Ann}_{\mathscr{A}}(L) \subset \mathscr{J}$. We further claim that for any $\mathrm{F}, \mathrm{G} \in$ $\mathcal{L} \cap \mathscr{C}(i, j)$ we even have

$$
\operatorname{Hom}_{\mathscr{J}^{\prime}(i, j)}(\mathrm{F}, \mathrm{G})=\operatorname{Hom}_{\mathscr{J}(i, j)}(\mathrm{F}, \mathrm{G}) .
$$

Indeed, by [MM, Lemma 21], $\operatorname{Hom}_{\mathscr{A}(\mathrm{i}, \mathrm{j})}(\mathrm{F}, \mathrm{G}) / \operatorname{Hom}_{\mathscr{J}^{\prime}}(\mathrm{F}, \mathrm{G})$ is mapped bijectively onto the space $\operatorname{Hom}_{\mathbf{C}_{\mathcal{L}}(j)}\left(\mathrm{F} L_{\mathrm{G}_{\mathcal{L}}}, \mathrm{G} L_{\mathrm{G}_{\mathcal{L}}}\right)$, so, by [MM, Lemma 19], the codimension of the left space in $\operatorname{Hom}_{\mathscr{A}(i, j)}(\mathrm{F}, \mathrm{G})$ equals the multiplicity $m$ of $\mathrm{G}_{\mathcal{L}}$ in $\mathrm{G}^{*} \circ \mathrm{F}$.

On the other hand, using adjunction, we obtain

$$
\begin{aligned}
\operatorname{Hom}_{\mathbf{D}_{\mathcal{L}}(\mathrm{j})}(\mathrm{F}, \mathrm{G}) & =\operatorname{Hom}_{\left(\mathbb{P}_{\mathrm{i}}^{\mathscr{A}} / \mathscr{J}\right)(\mathrm{j})}(\mathrm{F}, \mathrm{G}) \\
& \cong \operatorname{Hom}_{\left(\mathbb{P}_{\mathrm{i}}^{\mathscr{A}} / \mathscr{J}\right)(\mathrm{i})}\left(\mathrm{G}^{*} \circ \mathrm{F}, \mathbb{1}_{\mathrm{i}}\right) .
\end{aligned}
$$

By [MM, Proposition $17(\mathrm{a})]$, there is $0 \neq \alpha \in \operatorname{Hom}_{\mathbb{P}_{\mathrm{i}}^{\mathscr{A}}(\mathrm{i})}\left(\mathrm{G}_{\mathcal{L}}, \mathbb{1}_{\mathrm{i}}\right)$ such that the 2-morphism $\mathrm{F}(\alpha): \mathrm{F} \circ \mathrm{G}_{\mathcal{L}} \rightarrow \mathrm{F}$ is an epimorphism for any $\mathrm{F} \in \mathcal{L}$, so $F$ appears as a direct summand of $\mathrm{F} \circ \mathrm{G}_{\mathcal{L}}$. If $\mathscr{J}$ contained $\alpha$, being a left 2 -ideal, $\mathscr{J}$ would contain $\mathrm{F}(\alpha)$ as well, and hence, composing it with an inclusion from a direct summand, $\mathscr{J}$ would contain $\mathrm{id}_{\mathrm{F}}$, a contradiction. Hence $\alpha \notin \mathscr{J}$, which yields $\operatorname{Hom}_{\left(\mathbb{P}_{\mathrm{i}}^{\mathscr{A}} / \mathscr{J}\right)(\mathrm{i})}\left(\mathrm{G}_{\mathcal{L}}, \mathbb{1}_{\mathrm{i}}\right) \neq 0$, so $\operatorname{dim} \operatorname{Hom}_{\left(\mathbb{P}_{\mathrm{i}}^{\mathscr{A}} / \mathscr{J}\right)(\mathrm{i})}\left(\mathrm{G}^{*} \circ \mathrm{F}, \mathbb{1}_{\mathrm{i}}\right) \geq m$, and thus

$$
\operatorname{dim} \operatorname{Hom}_{\mathbf{D}_{\mathcal{L}}(\mathrm{j})}(\mathrm{F}, \mathrm{G}) \geq m=\operatorname{dim} \operatorname{Hom}_{\mathbf{C}_{\mathcal{L}}(\mathrm{j})}\left(\mathrm{F} L_{\mathrm{G}_{\mathcal{L}}}, \mathrm{G} L_{\mathrm{G}_{\mathcal{L}}}\right) .
$$

Taking codimensions in $\operatorname{Hom}_{\mathscr{A}(i, j)}(\mathrm{F}, \mathrm{G})$, this implies

$$
\operatorname{dim} \operatorname{Hom}_{\mathscr{J}(i, j)}(F, G) \leq \operatorname{dim} \operatorname{Hom}_{\mathscr{J}^{\prime}(i, j)}(F, G)
$$

and hence proves (3). It follows that $\Phi$ factors over $\mathbb{P}_{i}^{\mathscr{A}} / \mathscr{J}$ and restricts to an elementary equivalence from $\mathbf{D}_{\mathcal{L}}$ to $\mathbf{C}_{\mathcal{L}}$.

\section{Further EXAMPLES AND CONSTRUCTIONS}

7.1. $\mathfrak{s l}_{2}$-categorification. Fix $n \in\{0,1,2, \ldots\}$. Denote by $\mathrm{C}_{n}$ the complex coinvariant algebra of the symmetric group $S_{n}$, that is the quotient $\mathbb{C}\left[x_{1}, x_{2}, \ldots, x_{n}\right] / I$, where the ideal $I$ is generated by all homogeneous $S_{n}$-symmetric polynomials of positive degree. For $i=1,2, \ldots, n-1$ let $s_{i}$ denote the transposition $(i, i+1)$ and $S_{n}^{i}$ the subgroup of $S_{n}$ generated by all $s_{j}, j \neq i$. Set $\mathrm{C}_{n}^{0}=\mathrm{C}_{n}^{n}:=\mathbb{C}$ and for $i=1,2, \ldots, n-1$ define $\mathrm{C}_{n}^{i}$ as the subalgebra of $S_{n}^{i}$-invariants in $\mathrm{C}_{n}$ (note that $\left.\operatorname{dim} \mathrm{C}_{n}^{i}=\left(\begin{array}{c}n \\ i\end{array}\right)\right)$. Similarly, for different $i, j$ we denote by $S_{n}^{i, j}$ the subgroup of $S_{n}$ generated by all $s_{k}, k \neq i, j$, and define $\mathrm{C}_{n}^{i, j}$ to be the subalgebra $S_{n}^{i, j}$-invariants 
in $\mathrm{C}_{n}$. It is well known that both $\mathrm{C}_{n}, \mathrm{C}_{n}^{i}$ and $\mathrm{C}_{n}^{i, j}$ are symmetric algebras and that $\mathrm{C}_{n}^{i, i+1}$ is free both as a $\mathrm{C}_{n}^{i}$ - and $\mathrm{C}_{n}^{i+1}$-module (this follows from the geometric interpretation of the coinvariant algebra as the cohomology algebra of a flag variety, see e.g $[\mathrm{Hi}])$.

Define the 2-category $\mathscr{B}_{n}$ via its defining representation as follows: The objects of $\mathscr{B}_{n}$ are $0,1, \ldots, \mathrm{n}$ where we identify the object $\mathrm{i}$ with some small category $\mathcal{C}_{i}$ equivalent to the category of $\mathrm{C}_{n}^{i}$-modules. As generating 1-morphisms between $\mathcal{C}_{i}$ and $\mathcal{C}_{i+1}$ we take the functors

(4)

$\mathrm{F}_{i}^{(1)}:=\operatorname{Res}_{\mathrm{C}_{n}^{i+1}}^{\mathrm{C}_{n}^{i, i+1}} \circ \operatorname{Ind}_{\mathrm{C}_{n}^{i}}^{\mathrm{C}_{n}^{i, i+1}}: \mathcal{C}_{i} \rightarrow \mathcal{C}_{i+1}$ and $\mathrm{E}_{i+1}^{(1)}:=\operatorname{Res}_{\mathrm{C}_{n}^{i}}^{\mathrm{C}_{n}^{i, i+1}} \circ \operatorname{Ind}_{\mathrm{C}_{n}^{i+1}} \mathrm{C}^{\mathrm{C}_{n}^{i, i+1}} \mathcal{C}_{i+1} \rightarrow \mathcal{C}_{i}$.

We let $\mathscr{B}_{n}$ be the minimal full fully additive 2 -subcategory of the endomorphism 2 -category of the $\mathcal{C}_{i}$ 's containing these generating functors and closed with respect to isomorphism of 1-morphisms. Thus, 2-morphisms in $\mathscr{B}_{n}$ are all natural transformations of functors.

Remark 23. The 2-category $\mathscr{B}_{n}$ has an alternative description via the BGG category $\mathcal{O}$ for $\mathfrak{g l}_{n}$. Let $\mathfrak{g l}_{n}=\mathfrak{n}_{-} \oplus \mathfrak{h} \oplus \mathfrak{n}_{+}$be the standard triangular decomposition of $\mathfrak{g l}_{n}$. Fix a basis $\left\{e_{i i}\right\} \in \mathfrak{h}$ and the corresponding dual basis in $\mathfrak{h}^{*}$. Denote by $\mathcal{O}^{i}$ the block of the category $\mathcal{O}$ associated to this triangular decomposition (see [BGG, Hu]) containing simple highest weight modules $L(\lambda)$ (with highest weight $\lambda-\rho$ where $\rho$ is the half sum of all positive roots), where $\lambda \in \mathfrak{h}^{*}$ is a $0-1$ vector (with respect to the basis chosen above) with exactly $i$ components equal to 0 . The block $\mathcal{O}^{i}$ has a unique (up to isomorphism) indecomposable projective-injective module, which we denote by $P_{i}$, and $\operatorname{End}_{\mathcal{O}}\left(P_{i}\right) \cong \mathrm{C}_{n}^{i}$. The full subcategory $\mathcal{P}_{i}$ of $\mathcal{O}^{i}$ consisting of all modules with projective-injective presentation is then equivalent to $\mathrm{C}_{n}^{i}$-mod. This establishes a connection to $\mathscr{B}_{n}$ (one has to choose appropriate small versions of all $\mathcal{P}_{i}$ 's). Generating 1-morphisms from (4) are realized via projective functors between different blocks of $\mathcal{O}$ in the sense of [BG] (in this case these are just tensoring with the natural representation of $\mathfrak{g l}_{n}$ or its dual, followed by the projection onto an appropriate block). We refer to [BFK, FKS] for further details.

Remark 24. Some other versions of $\mathscr{B}_{n}$ can be found implicitly in [CR, La] (and some other papers). One essential difference with all these papers is that they also prescribe generating 2-morphisms while we just take all of them (following $[\mathrm{BFK}]$ ).

Let $i \in\{0,1, \ldots, n\}$ and $k, l \in\{0,1, \ldots, n\}$ be such that $i+k, i-l \in\{0,1, \ldots, n\}$. Similarly to the above define

$\mathrm{F}_{i}^{(k)}:=\operatorname{Res}_{\mathrm{C}_{n}^{i+k}}^{\mathrm{C}_{n}^{i, i+k}} \circ \operatorname{Ind}_{\mathrm{C}_{n}^{i}}^{\mathrm{C}_{n}^{i, i+k}}: \mathcal{C}_{i} \rightarrow \mathcal{C}_{i+k}$ and $\mathrm{E}_{i+k}^{(k)}:=\operatorname{Res}_{\mathrm{C}_{n}^{i}}^{\mathrm{C}_{n}^{i, i+k}} \circ \operatorname{Ind}_{\mathrm{C}_{n}^{i+k}}^{\mathrm{C}_{n}^{i, i+k}}: \mathcal{C}_{i+k} \rightarrow \mathcal{C}_{i}$

It is convenient to write $\mathrm{F}_{i}^{(0)}$ and $\mathrm{E}_{i}^{(0)}$ for $\mathbb{1}_{i}$. From the facts that $\mathrm{C}_{n}, \mathrm{C}_{n}^{i}$ and $\mathrm{C}_{n}^{i, j}$ are symmetric and that $\mathrm{C}_{n}^{i, i+1}$ is free both as a $\mathrm{C}_{n}^{i}$ - and $\mathrm{C}_{n}^{i+1}$-module it follows that the functor $\mathrm{F}_{i}^{(k)}$ is both left and right adjoint (i.e. biadjoint) to $\mathrm{E}_{i+k}^{(k)}$. Indecomposable 1 -morphisms in $\mathscr{B}_{n}$ are given by the following claim proved in [BFK, 3.1.3]:

Proposition 25. Let $i, j \in\{0,1, \ldots, n\}$. The following is a complete and irredundant list of indecomposable 1-morphisms in $\mathscr{B}_{n}(\mathrm{i}, \mathrm{j})$, up to isomorphism:

(a) $\mathrm{F}_{i}^{(j-i)}, \mathrm{F}_{i+1}^{(j-i+1)} \mathrm{E}_{i}^{(1)}, \mathrm{F}_{i+2}^{(j-i+2)} \mathrm{E}_{i}^{(2)}, \ldots, \mathrm{F}_{0}^{(j)} \mathrm{E}_{i}^{(i)}$, if $i<j$ and $i<n-j$.

(b) $\mathrm{F}_{i}^{(j-i)}, \mathrm{E}_{j+1}^{(1)} \mathrm{F}_{i}^{(j-i+1)}, \mathrm{E}_{j+2}^{(2)} \mathrm{F}_{i}^{(j-i+2)}, \ldots, \mathrm{E}_{n}^{(n-j)} \mathrm{F}_{i}^{(n-i)}$, if $i<j$ and $i \geq n-j$.

(c) $\mathrm{E}_{i}^{(i-j)}, \mathrm{E}_{i+1}^{(i-j+1)} \mathrm{F}_{i}^{(1)}, \mathrm{E}_{i+2}^{(i-j+2)} \mathrm{F}_{i}^{(2)}, \ldots, \mathrm{E}_{n}^{(n-j)} \mathrm{F}_{i}^{(n-i)}$, if $i>j$ and $j \geq n-i$. 
(d) $\mathrm{E}_{i}^{(i-j)}, \mathrm{F}_{j-1}^{(1)} \mathrm{E}_{i}^{(i-j+1)}, \mathrm{F}_{j-2}^{(2)} \mathrm{E}_{i}^{(i-j+2)}, \ldots, \mathrm{F}_{0}^{(j)} \mathrm{E}_{i}^{(i)}$, if $i>j$ and $i<n-j$.

(e) $\mathbb{1}_{\mathrm{i}}, \mathrm{F}_{i-1}^{(1)} \mathrm{E}_{i}^{(1)}, \mathrm{F}_{i-2}^{(2)} \mathrm{E}_{i}^{(2)}, \ldots, \mathrm{F}_{0}^{(i)} \mathrm{E}_{i}^{(i)}$, if $i=j$ and $i<n-i$.

(f) $\mathbb{1}_{\mathrm{i}}, \mathrm{E}_{i+1}^{(1)} \mathrm{F}_{i}^{(1)}, \mathrm{E}_{i+2}^{(2)} \mathrm{F}_{i}^{(2)}, \ldots, \mathrm{E}_{n}^{(n-i)} \mathrm{F}_{n-i}^{(n-i)}$, if $i=j$ and $i \geq n-i$.

Corollary 26. The 2-category $\mathscr{B}_{n}$ is fiat.

Proof. From Proposition 25 we see that $\mathscr{B}_{n}$ has only finitely many indecomposable 1-morphisms up to isomorphism. The weak involution is given by taking the adjoint functor, which also implies existence of adjunction morphisms. The rest is clear.

Set $\mathrm{E}_{0}^{(1)}=\mathrm{E}_{n+1}^{(1)}=\mathrm{F}_{-1}^{(1)}=\mathrm{F}_{n}^{(1)}=0$. Then [BFK, Theorem 1] asserts that for every $i \in\{0,1, \ldots, n\}$ there is an isomorphism of functors as follows:

$$
\left(\mathrm{E}_{i+1}^{(1)} \circ \mathrm{F}_{i}^{(1)}\right) \oplus \mathbb{1}_{\mathbf{i}}^{\oplus(i)} \cong\left(\mathrm{F}_{i-1}^{(1)} \circ \mathrm{E}_{i+1}^{(1)}\right) \oplus \mathbb{1}_{\mathbf{i}}^{\oplus(n-i)} .
$$

Furthermore, for every $i \in\{0,1, \ldots, n\}, k=1,2, \ldots, i$ and $l=1,2, \ldots, n-i$ there are isomorphisms of functors as follows:

(6) $\mathrm{E}_{i+(k-1)}^{(1)} \circ \cdots \circ \mathrm{E}_{i+1}^{(1)} \circ \mathrm{E}_{i}^{(1)} \cong\left(\mathrm{E}_{i}^{(k)}\right)^{\oplus k !}, \quad \mathrm{F}_{i-(k-1)}^{(1)} \circ \cdots \circ \mathrm{F}_{i-1}^{(1)} \circ \mathrm{F}_{i}^{(1)} \cong\left(\mathrm{F}_{i}^{(k)}\right)^{\oplus k !}$.

This can be used to describe cells in $\mathscr{B}_{n}$ as follows:

- for $i \in\left\{0,1, \ldots,\left\lfloor\frac{n}{2}\right\rfloor\right\}$ let $\mathcal{J}_{i}$ denote the two-sided cell of $\mathscr{B}_{n}$ containing $\mathbb{1}_{i}$;

- for $(i, k)$ such that $i \in\left\{0,1, \ldots\left\lfloor\frac{n}{2}\right\rfloor\right\}$ and $k \in\{0,1, \ldots, i\}$ let $\mathcal{L}_{(i, k)}$ denote the left cell of $\mathscr{B}_{n}$ containing $\mathrm{F}_{i-k}^{(k)} \mathrm{E}_{i}^{(k)}$;

- for $(i, k)$ such that $i \in\left\{\left\lfloor\frac{n}{2}\right\rfloor+1, \ldots, n\right\}$ and $k \in\{0,1, \ldots, n-i\}$ let $\mathcal{L}_{(i, k)}$ denote the left cell of $\mathscr{B}_{n}$ containing $\mathrm{E}_{i+k}^{(k)} \mathrm{F}_{i}^{(k)}$;

- for $(i, k)$ such that $i \in\left\{0,1, \ldots\left\lfloor\frac{n}{2}\right\rfloor\right\}$ and $k \in\{0,1, \ldots, i\}$ let $\mathcal{R}_{(i, k)}$ denote the right cell of $\mathscr{B}_{n}$ containing $\mathrm{F}_{i-k}^{(k)} \mathrm{E}_{i}^{(k)}$;

- for $(i, k)$ such that $i \in\left\{\left\lfloor\frac{n}{2}\right\rfloor+1, \ldots, n\right\}$ and $k \in\{0,1, \ldots, n-i\}$ let $\mathcal{R}_{(i, k)}$ denote the right cell of $\mathscr{B}_{n}$ containing $\mathrm{E}_{i+k}^{(k)} \mathrm{F}_{i}^{(k)}$.

Proposition 27. (i) The two-sided cells $\mathcal{J}_{i}$ defined above give a complete and irredundant list of two-sided cells.

(ii) The left cells $\mathcal{L}_{(i, k)}$ defined above give a complete and irredundant list of left cells.

(iii) The right cells $\mathcal{R}_{(i, k)}$ defined above give a complete and irredundant list of right cells.

(iv) All two-sided cells of $\mathscr{B}_{n}$ are strongly regular and satisfy condition (2).

Proof. Claims (i)-(iii) follow from Proposition 25 and isomorphisms (5) and (6) by direct calculation. Similarly one checks that all two-sided cells of $\mathscr{B}_{n}$ are strongly regular, so we are only left to check condition (2).

Consider $\mathcal{R}_{(i, k)}$ for some fixed pair $(i, k)$. Assume $i \in\left\{0,1, \ldots\left\lfloor\frac{n}{2}\right\rfloor\right\}$ and $k \in$ $\{0,1, \ldots, i\}$ (the other case is dealt with by a similar argument). Then, using 
Proposition 25 and isomorphisms (5) and (6) one shows that $\mathcal{R}_{(i, k)}$ consists exactly of the following morphisms (here $s:=\left\lfloor\frac{n}{2}\right\rfloor$ ):

$$
\begin{gathered}
\mathrm{F}_{k}^{(i-k)}, \mathrm{F}_{k}^{(i-k)} \mathrm{E}_{k+1}^{(1)}, \ldots, \mathrm{F}_{k}^{(i-k)} \mathrm{E}_{s}^{(s-k)}, \\
\mathrm{E}_{n-k}^{(n-k-i)}, \mathrm{E}_{n-k}^{(n-k-i)} \mathrm{F}_{n-k-1}^{(1)}, \ldots, \mathrm{E}_{n-k}^{(n-k-i)} \mathrm{F}_{s+1}^{(n-k-(s+1))} .
\end{gathered}
$$

Using (5) one checks that the value of $m_{\mathrm{F}}$ on all these 1-morphisms equals the multiplicity of $\mathbb{1}_{\mathrm{k}}$ in $\mathrm{E}_{i}^{(i-k)} \mathrm{F}_{k}^{(i-k)}$.

Corollary 28. The defining representation of $\mathscr{B}_{n}$ is equivalent to $\overline{\mathbf{C}}_{\mathcal{L}_{(0,0)}}$.

Proof. Denote the defining representation of $\mathscr{B}_{n}$ by $\mathbf{M}$. Sending $\mathbb{1}_{0}$ to the projective simple module in $\mathcal{C}_{0}$ restricts to a homomorphism from $\mathbf{C}_{\mathcal{L}_{(0,0)}}$ to $\mathbf{M}_{p r}$ which is easily seen to be an elementary equivalence. The claim now follows from Theorem 11.

It is easy to check that the decategorification of $\overline{\mathbf{C}}_{\mathcal{L}_{(0,0)}}$ gives a simple $n+1$ dimensional $\mathfrak{s l}_{2}$-module (the decategorification of $\mathscr{B}_{n}$ gives the image of Lusztig's idempotent completion $\dot{U}_{\mathfrak{s l}_{2}}$ of $U\left(\mathfrak{s l}_{2}\right)$ acting on this simple $n+1$-dimensional $\mathfrak{s l}_{2}$ module). All this is spelled out e.g. in [BFK, FKS].

Corollary 29. The 2-category $\mathscr{B}_{n}$ is $\mathcal{J}_{0}$-simple.

Proof. This follows from Corollary 28 and Theorem 19 as the defining representation is obviously faithful.

The latter implies the following recursion for $\mathscr{B}_{n}$ (for appropriate choices of the $\mathcal{C}_{i}$ 's in the corresponding defining representations):

Theorem 30. The 2-categories $\mathscr{B}_{n-2}$ and $\mathscr{B}_{n} / \operatorname{Ker}\left(\mathbf{C}_{\mathcal{L}_{1}}\right)$ are isomorphic.

Proof. We have natural surjective homomorphisms of algebras $\mathrm{C}_{n} \rightarrow \mathrm{C}_{n-2}$ given by forgetting $s_{1}$ and $s_{n-1}$, evaluating $x_{1}$ and $x_{n}$ at 0 , and sending other $x_{i}$ to $x_{i-1}$. The gives rise to surjections for all corresponding subalgebras of invariants. For appropriate choices of the $\mathcal{C}_{i}$ 's this induces a surjective 2 -functor $\Phi: \mathscr{B}_{n} \rightarrow \mathscr{B}_{n-2}$. By Corollary $29, \mathscr{B}_{n-2}$ is $\mathcal{J}_{0}$-simple (for its index 0 that corresponds to index 1 for the 2-category $\left.\mathscr{B}_{n}\right)$, which implies that the 2-ideal $\operatorname{Ker}\left(\mathbf{C}_{\mathcal{L}_{1}}\right)$ of $\mathscr{B}_{n}$ coincides with $\operatorname{Ker}(\Phi)$. The claim follows.

Remark 31. The ideal $\operatorname{Ker}\left(\mathbf{C}_{\mathcal{L}_{1}}\right)$ obviously contains the 2-morphism $\mathrm{id}_{\mathbb{1}_{0}}$ and hence also $i d_{F}$ for any $F$ in the 2 -sided cell of $\mathbb{1}_{0}$. However, it is easy to check that $\operatorname{Ker}\left(\mathbf{C}_{\mathcal{L}_{1}}\right)$ is not generated by $\mathrm{id}_{\mathbb{1}_{0}}$ in general.

7.2. 2-Schur algebra. Let $n, r \in \mathbb{N}$. Consider the Lie algebra $\mathfrak{g l}_{r}$ with a fixed standard triangular decomposition $\mathfrak{n}_{-} \oplus \mathfrak{h} \oplus \mathfrak{n}_{+}$. For $\lambda \in \mathfrak{h}^{*}$ let $M(\lambda)$ denote the Verma modules with highest weight $\lambda-\rho$, where $\rho$ is the half of the sum of all positive roots. For a dominant $\lambda \in \mathfrak{h}^{*}$ let $\mathcal{O}_{\lambda}$ be the block of the BGG category $\mathcal{O}$ ([BGG]) associated with this triangular decomposition, containing $M(\lambda)$, see $[\mathrm{Ma}]$ for details.

We fix in $\mathfrak{h}^{*}$ the basis, dual to the basis of $\mathfrak{h}$ consisting of matrix units. Using this basis, we identify $\mathfrak{h}^{*}$ with $\mathbb{C}^{r}$. Denote by $\mathrm{N}$ the set of all vectors $\mathbf{v}:=\left(v_{1}, v_{2}, \ldots, v_{r}\right) \in \mathbb{C}^{r}$ such that $v_{i} \in\{1,2, \ldots, n\}$ for all $i$. Let $\mathrm{N}_{d}$ denote the subset of $\mathrm{N}$ consisting of all dominant vectors, that is all $\left(v_{1}, v_{2}, \ldots, v_{r}\right)$ such that $v_{1} \geq v_{2} \geq \cdots \geq v_{r}$. 
Consider the 2-category $\mathscr{D}_{n, r}$ defined via its defining representation as follows: objects of $\mathscr{D}_{n, r}$ are $\mathbf{v} \in \mathrm{N}_{d}$, which we identify with some small category $\mathcal{Q}_{\mathbf{v}}$, equivalent to $\mathcal{O}_{\mathbf{v}} ; 1$-morphisms are projective functors in the sense of $[\mathrm{BG}]$ and 2-morphisms are natural transformations of functors. This is a fiat category, see [MM, 7.2]. By [MS1, Section 9], the decategorification of the defining representation of $\mathscr{D}_{n, r}$ gives the defining representation of the Schur algebra $S(n, r)$ (see also [MS2]). This allows us to consider $\mathscr{D}_{n, r}$ as a categorification (a 2-analogue) of $S(n, r)$ and here we describe the combinatorics of $\mathscr{D}_{n, r}$ in more details. We refer the reader to [Mat] for more details on the Schur algebra associated to the symmetric group.

For $\mathbf{v} \in \mathrm{N}_{d}$ let $\mathrm{N}_{\mathbf{v}}$ and $S_{\mathbf{v}}$ denote the orbit of $\mathbf{v}$ under the action of the symmetric group $S_{r}$ and the stabilizer of $\mathbf{v}$ with respect to this action, respectively. By $[\mathrm{BG}]$, for $\mathbf{v}, \mathbf{u} \in \mathrm{N}_{d}$ indecomposable projective functors from $\mathcal{Q}_{\mathbf{v}}$ to $\mathcal{Q}_{\mathbf{u}}$ correspond bijectively to $\mathbf{v}$-antidominant elements in $\mathrm{N}_{\mathbf{u}}$ (that is $\mathbf{x} \in \mathrm{N}_{\mathbf{u}}$ such that for any $i \in\{1,2, \ldots, r-1\}$ the equality $v_{i}=v_{i+1}$ implies the inequality $x_{i} \leq x_{i+1}$ ), alternatively, to double cosets $S_{\mathbf{v}} \backslash S_{r} / S_{\mathbf{u}}$. Moreover, by [BG] the decategorifications of indecomposable projective functors are linearly independent. As the indexing set for indecomposable projective functors coincides with the indexing set of the standard basis for $S(n, r)$, we conclude that the decategorification of $\mathscr{D}_{n, r}$ is isomorphic to $S(n, r)$. The distinguished basis of $S(n, r)$ corresponding to indecomposable projective functors, given by this construction, is Du's canonical basis of $S(n, r)$ defined in $[\mathrm{Du}]$.

The Robinson-Schensted-Knuth algorithm (see $[\mathrm{Kn}]$ ) provides a bijection between the set

$$
\left\{(\mathbf{v}, \mathbf{u}): \mathbf{v}, \mathbf{u} \in \mathrm{N}_{d}, \mathbf{u} \text { is } \mathbf{v} \text {-antidominant }\right\}
$$

(which indexes indecomposable projective functors) and the set of all pairs $(\alpha, \beta)$ of semistandard Young tableaux of the same shape and content from $\{1,2, \ldots, n\}$. For an indecomposable 1-morphism $\theta$ in $\mathscr{D}_{n, r}$ we denote by $\left(\alpha_{\theta}, \beta_{\theta}\right)$ the corresponding pair of semistandard Young tableaux.

Theorem 32. Let $\theta$ and $\theta^{\prime}$ be two indecomposable 1-morphisms in $\mathscr{D}_{n, r}$.

(i) We have $\theta \sim_{\mathcal{L}} \theta^{\prime}$ if and only if $\alpha_{\theta}=\alpha_{\theta^{\prime}}$.

(ii) We have $\theta \sim_{\mathcal{R}} \theta^{\prime}$ if and only if $\beta_{\theta}=\beta_{\theta^{\prime}}$.

(iii) All two-sided cells in $\mathscr{D}_{n, r}$ are strongly regular and satisfy (2).

Proof. All claims reduce to the corresponding statements for the regular block of $\mathcal{O}$, see $[\mathrm{MM}, 7.1]$, by first translating out of the source wall of the projective functor and then back onto the target wall.

7.3. Image completion. Let $\mathscr{C}$ be a fiat 2-category and $\mathbf{M} \in \mathscr{C}$-mod. Define a new 2-category $\mathscr{A}$ as follows: objects of $\mathscr{A}$ are the same as objects of $\mathscr{C}$; for $i, j \in \mathscr{A}, 1$-morphisms in $\mathscr{A}(i, j)$ are all functors from $\mathbf{M}(i)$ to $\mathbf{M}(j)$, isomorphic to $\mathbf{M}(\mathrm{F})$, where $\mathrm{F} \in \mathscr{C}(i, j) ; 2$-morphisms in $\mathscr{A}$ are all natural transformations of functors; the composition in $\mathscr{A}$ is given by the usual composition of functors. The 2-category $\mathscr{A}$ will be called the completion of $\mathbf{M}(\mathscr{C})$.

Proposition 33. The 2-category $\mathscr{A}$ is weakly fiat in the sence that it has all properties of a fiat 2-category with one exception that the weak anti-autoequivalence * does not have to be involutive. 
Proof. Since $\mathbf{M} \in \mathscr{C}$-mod and each $\mathbf{M}(\mathrm{F})$ is exact, each $\mathbf{M}(\mathrm{F})$ decomposes into a finite number of indecomposable functors. This implies that $\mathscr{A}$ has finitely many indecomposable 1-morphisms (up to isomorphism). Clearly, all spaces of 2-morphisms are finite dimensional over $\mathbb{k}$. Now the claim follows from the standard fact (see e.g. $[B G, 1.2(\mathrm{c})])$ that if some functor $\mathrm{X}: \mathbf{M}(\mathrm{i}) \rightarrow \mathbf{M}(\mathrm{j})$ has a right adjoint $\mathrm{Y}: \mathbf{M}(\mathrm{j}) \rightarrow \mathbf{M}(\mathrm{i})$, then every direct summand of $\mathrm{X}$ has a right adjoint which is a direct summand of $\mathrm{Y}$.

Remark 34. There is no obvious relation between $\mathscr{A}$ and $\mathscr{C}$. For instance, $\mathscr{A}$ can have many more 2-morphisms, in particular, new idempotent 2-morphisms, and thus many more indecomposable 1-morphisms. The other extreme is that we can map different 1-morphisms to the same thing making 2-morphisms composable in $\mathscr{A}$ which were not composable in $\mathscr{C}$.

\section{REFERENCES}

[Ag] T. Agerholm; Simple 2-representations and classification of categorifications. PhD Thesis, Aarhus University, 2011.

[BFK] J. Bernstein, I. Frenkel, M. Khovanov; A categorification of the Temperley-Lieb algebra and Schur quotients of $U\left(\mathfrak{s l}_{2}\right)$ via projective and Zuckerman functors. Selecta Math. (N.S.) 5 (1999), no. 2, 199-241.

[BGG] J. Bernstein, I. Gelfand, S. Gelfand; A certain category of $\mathfrak{g}$-modules. Funkcional. Anal. i Priložen. 10 (1976), no. 2, 1-8.

[BG] J. Bernstein, S. Gelfand; Tensor products of finite- and infinite-dimensional representations of semisimple Lie algebras. Compositio Math. 41 (1980), no. 2, 245-285.

[CR] J. Chuang, R. Rouquier; Derived equivalences for symmetric groups and $\mathfrak{s l}_{2}$-categorification. Ann. of Math. (2) 167 (2008), no. 1, 245-298.

[Du] J. Du; Kazhdan-Lusztig bases and isomorphism theorems for $q$-Schur algebras. KazhdanLusztig theory and related topics (Chicago, IL, 1989), 121-140, Contemp. Math., 139, Amer. Math. Soc., Providence, RI, 1992.

[FKS] I. Frenkel, M. Khovanov, C. Stroppel; A categorification of finite-dimensional irreducible representations of quantum $\mathfrak{s l}_{2}$ and their tensor products. Selecta Math. (N.S.) 12 (2006), no. 3-4, 379-431.

[Fr] P. Freyd; Representations in abelian categories. in: Proc. Conf. Categorical Algebra (1966), 95-120.

[Gr] J. Green; On the structure of semigroups. Ann. of Math. (2) 54, (1951). 163-172.

[Hi] H. Hiller; Geometry of Coxeter groups. Research Notes in Mathematics, 54. Pitman (Advanced Publishing Program), Boston, Mass.-London, 1982.

$[\mathrm{Hu}] \quad$ J. Humphreys; Representations of semisimple Lie algebras in the BGG category $\mathcal{O}$. Graduate Studies in Mathematics, 94. American Mathematical Society, Providence, RI, 2008.

[Kn] D. Knuth; Permutations, matrices, and generalized Young tableaux. Pacific J. Math. 234 (1970), 709-727.

[La] A. Lauda; A categorification of quantum $\mathfrak{s l}(2)$. Adv. Math. 225 (2010), no. 6, 3327-3424.

[McL] S. Mac Lane; Categories for the working mathematician. Second edition. Graduate Texts in Mathematics, 5. Springer-Verlag, New York, 1998.

[Mat] A. Mathas; Iwahori-Hecke algebras and Schur algebras of the symmetric group. University Lecture Series, 15. American Mathematical Society, Providence, RI, 1999.

[Ma] V. Mazorchuk; Lectures on algebraic categorification, QGM Master Class Series. European Mathematical Society (EMS), Zürich, 2012.

[MM] V. Mazorchuk, V. Miemietz; Cell 2-representations of finitary 2-categories; Compositio Math. 147 (2011), 1519-1545.

[MS1] V. Mazorchuk, C. Stroppel; Categorification of (induced) cell modules and the rough structure of generalised Verma modules. Adv. Math. 219 (2008), no. 4, 1363-1426.

[MS2] V. Mazorchuk, C. Stroppel; A combinatorial approach to functorial quantum $\mathfrak{s l}_{k}$ knot invariants. Amer. J. Math. 131 (2009), no. 6, 1679-1713.

[Os] V. Ostrik; Tensor ideals in the category of tilting modules. Transform. Groups 2 (1997), no. 3, 279-287.

[Ro1] R. Rouquier; Categorification of the braid groups. Preprint arXiv:math/0409593.

[Ro2] R. Rouquier; 2-Kac-Moody algebras. Preprint arXiv:0812.5023. 
[So] W. Soergel; The combinatorics of Harish-Chandra bimodules. J. Reine Angew. Math. 429 (1992), 49-74.

[Vi] O. Viro; Hyperfields for Tropical Geometry I. Hyperfields and dequantization. Preprint arXiv:1006.3034.

Volodymyr Mazorchuk, Department of Mathematics, Uppsala University, Box 480, 751 06, Uppsala, SWEDEN, mazor@math .uu . se; http://www.math.uu.se/\%mazor/.

Vanessa Miemietz, School of Mathematics, University of East Anglia, Norwich, UK, NR4 7TJ, v.miemietz@uea.ac.uk; http://www.uea.ac.uk//byr09xgu/. 